Article

\title{
Typology of Pure Deodar Forests Driven by Vegetation-Environment Relations in Manoor Valley, Northwestern Himalaya
}

\author{
Inayat Ur Rahman 1,2 ${ }^{1}$, Aftab Afzal ${ }^{1} \mathbb{D}$, Zafar Iqbal ${ }^{1}$, Eduardo Soares Calixto ${ }^{3,4} \mathbb{D}$, Jawaher Alkahtani ${ }^{5}$, \\ Mona S. Alwahibi ${ }^{5}$, Niaz Ali ${ }^{1, *} \mathbb{D}$, Rukhsana Kausar ${ }^{6}\left(\mathbb{D}\right.$, Uzma Khan ${ }^{1}$ and Rainer W. Bussmann ${ }^{7}(\mathbb{D}$
}

1 Department of Botany, Hazara University, Mansehra 21300, Pakistan; hajibotanist@outlook.com (I.U.R.); aftabafzalkiani@yahoo.com (A.A.); drzafar.hu@yahoo.com (Z.I.); uzmaqau2003@yahoo.com (U.K.)

2 William L. Brown Center, Missouri Botanical Garden, 4344 Shaw Blvd, St. Louis, MO 63110, USA

3 Department of Biological Sciences, University of Missouri, St. Louis, MO 63110, USA; calixtos.edu@gmail.com

4 Entomology and Nematology Department, University of Florida, Gainesville, FL 32611, USA

5 Department of Botany and Microbiology, College of Science, King Saud University, Riyadh 11451, Saudi Arabia; jsalkahatani@ksu.edu.sa (J.A.); malwahibi@ksu.edu.sa (M.S.A.)

6 Department of Environmental Sciences, International Islamic University, Islamabad 44000, Pakistan; rukhsana.tariq@iiu.edu.pk

7 Department of Ethnobotany, Institute of Botany, Ilia State University, 1 Botanical Street, Tbilisi 0105, Georgia; rainer.bussmann@iliauni.edu.ge

* Correspondence: niazalitk25@gmail.com

check for updates

Citation: Rahman, I.U.; Afzal, A.; Iqbal, Z.; Calixto, E.S.; Alkahtani, J.; Alwahibi, M.S.; Ali, N.; Kausar, R.; Khan, U.; Bussmann, R.W. Typology of Pure Deodar Forests Driven by Vegetation-Environment Relations in Manoor Valley, Northwestern

Himalaya. Appl. Sci. 2022, 12, 2753. https://doi.org/10.3390/ app12052753

Received: 24 August 2021 Accepted: 29 November 2021 Published: 7 March 2022

Publisher's Note: MDPI stays neutral with regard to jurisdictional claims in published maps and institutional affiliations.

Copyright: (c) 2022 by the authors. Licensee MDPI, Basel, Switzerland. This article is an open access article distributed under the terms and conditions of the Creative Commons Attribution (CC BY) license (https:// creativecommons.org/licenses/by/ $4.0 /)$.

\begin{abstract}
The current research was carried out to characterize the phytosociology of the forests of one of Pakistan's most valuable tree species (Deodar) across its native range. In this context, our main hypothesis was that, along the altitudinal gradient, we would find different plant communities that would be driven by different environmental variables (climatic, edaphic, and physiographic). Therefore, to assess the vegetation structure of the pure Deodar forests of the unexplored Manoor Valley (Northwestern Himalaya), Pakistan, frequent field visits were carried out during different seasons of 2015-2018. Ecological methods: Line transects sampling (23 stands) and phytosociological attributes were evaluated in relation to geographical and environmental variables. Various statistical software applications (i.e., PCORD, RStudio 4.0, and R 3.6.1) were used to examine all of the gathered data of plant species and environmental variables. A total of three different plant communities (Cedrus-Isodon-Cynodon, Cedrus-Cynodon-Dryopteris, and Sambucus-Cedrus-Desmodium) were identified by grouping 162 species and 23 stands in pure Deodar forests under the influence of geographic, slope, edaphic, and climatic variables, ranging from 1580.8 to $2373.8 \mathrm{~m}$. The altitude (1936-2373 m), slope angle $\left(25-85^{\circ}\right)$, sandy (29-48\%) and loamy soil texture, wind speed $\left(1.45 \mathrm{~ms}^{-1}\right)$, and temperature $\left(25.8^{\circ} \mathrm{C}\right)$ all had a strong influence on the Sambucus-Cedrus-Desmodium community. In contrast with this, the Cedrus-Cynodon-Dryopteris community showed a positively significant relationship with the northeastern slope, silty (32-58\%) and sandy (15.8-55\%) loamy soil texture, and barometric pressure (814.3 pa). Nonetheless, the Cedrus-Isodon-Cynodon community revealed a significant positive association with the northeastern to southwestern slope, $\mathrm{pH}$ (6.3), wet bulb (19.7), and dew point (17.7). We found significant differences $(p<0.001)$ among the three communities found in the pure Deodar forests in the four diversity indexes. The Sambucus-Cedrus-Desmodium community has the maximum number of plants (129 species), Shannon's diversity $\left(\mathrm{H}^{\prime}=3.7\right)$, and Simpson's dominance (0.98) values among the recorded communities. The Pielou's evenness index value was led by the Cedrus-Isodon-Cynodon community (0.97). Beta diversity showed a dissimilarity lower than $50 \%$ among the three communities. Simple term effects in the canonical correspondence analysis model revealed significant $(p<0.05)$ differences in altitude, slope angle, slope (southeastern), and wind speed variables. The present investigation sheds light on vegetation pattern and species contribution as a function of environmental gradients and provides a baseline for future studies.
\end{abstract}


Keywords: plants communities; pure Deodar forests; environmental variables; multivariate approaches; Himalaya

\section{Introduction}

The study of vegetation categorization based on species co-occurrence and its relationship to environmental factors is known as phytosociology [1,2]. This research area has resulted in major vegetation appraisal techniques and methodologies that may be linked to ecosystem services and biodiversity conservation [3]. Species composition, community structure, and function are the most significant ecological features of forest ecosystems, and they all change in response to environmental changes [4,5]. Factors such as vegetation type, slope, aspect, edaphic variables, and altitude [6,7] influence the community composition, structure, and distribution pattern of diversity in mountain vegetation [8]. Elevation is an important variable in mountain ecosystems [9] since it affects the structure of the vegetation in most mountains across the world [10]. Most of the environmental factors, as well as species diversity [11,12], change concurrently along the altitudinal gradient [13]. This aspect fosters habitat heterogeneity and induces micro-environmental alteration in vegetation patterning [14,15]. Heywood and Watson [16] reported that species richness in vascular plants increases with temperate latitude. Vegetation associations with ecological diversity are considered as a proportion of the quality of the entire ecosystem [17]. For this reason, vegetation in relation to environmental variables has been an important topic in recent years [18].

In Pakistan, Deodar (Cedrus deodara (Roxb. ex D.Don) G.Don) is found in Murree, Hazara, Abbottabad, Swat, Azad Kashmir, Kaghan Valley, Kohistan Chillas, Dir, and Chitral. It is mostly a dry temperate plant, although scattered stands of this species can be found in moist temperate regions [19]. A majority of these forests are found in the transitional zone between dry and moist temperate zones, with no clear boundary between the two [20]. Deodar grows on ridge tops and moderate $\left(17^{\circ}\right)$ to steep $\left(50^{\circ}\right)$ slopes in Northern areas, ranging in elevation from $1650 \mathrm{~m}$ (Kaghan) to $2927 \mathrm{~m}$ (Chitral) in moist temperate, dry temperate, and timberline regions [21]. According to Champion et al. [19], Cedrus deodara forests gradually extend into the dry inner valleys of the Himalayas. Hussain and Illahi [20] have compiled a comprehensive report on the ecology and vegetation types of Pakistan's lesser Himalaya. They further noted that Deodar trees cannot endure extremely moist environments and are thus restricted to the Himalayan drier zones.

Following the observational vegetation surveys by Champion et al. [19] and Beg [22], plenty of quantitative phytosociological studies have been conducted across the nation by several researchers. Ahmed et al. [23] carried out an extensive quantitative sampling in the Deodar forests of the Hindu Kush and Himalayan ranges of Pakistan, and they identified Cedrus deodara, Pinus wallichiana, P. gerardiana, and Abies pindrow as the dominant species in the recorded plant communities. Phytosociological work from Ayubia National Park [24] was reported three decades ago, and it was reported about a decade ago in the Sarsawa Hills, District Kotli of Azad Kashmir [25]. The former study reported five plant communities, i.e., Acacia modesta-Cannabis sativa, Acacia modesta-Cynodon dactylon, Acacia modesta-Themeda anathera, Acacia modesta-Dodonaea viscosa, and Acacia modesta-Lantana camara. The latter identified nine communities (i.e., Pinus-Poa-Maytenus, Myrsine-Themeda-Pinus, ColebrookiaThemeda-Dodonaea, Themeda-Carissa-Adhatoda, Themeda-Dodonaea-Eriophorum, AdhatodaThemeda, Carissa-Myrsine-Themeda, Carissa-Themeda-Dodonaea, and Dodonaea-Carissa-Pinus) at different elevations. The structure and distribution patterns of plant communities in Himalayan forests are poorly understood [26]. The Northwestern Himalaya is a unique bioregion because of its variable topographical and ecological complexity throughout a vast altitudinal range.

Nonetheless, apart from these studies, no thorough quantitative analyses have been conducted that define communities of Deodar forests, wherein the most valuable wood 
species reside, designating the Deodar as Pakistan's national tree. Therefore, the current research was carried out to characterize the phytosociology of the forests, owing to one of Pakistan's most valuable tree species across its native range. In this context, our main hypothesis was that, along the altitudinal gradient, we would find different plant communities that would be driven by different environmental variables (climatic, edaphic, and physiographic), due to changes in environmental factors. Overall, our study aims to move toward a better understanding of how environmental variables influence the structuring and distribution of plant species in the Deodar forest.

\section{Materials and Methods}

\subsection{Study Area}

The current field investigation was conducted in the pure Deodar forests of the Manoor valley, which is a hilly valley (Figure 1) in Pakistan's Northwestern Himalayan region [27-30], with elevations ranging from $1580.8 \mathrm{~m}$ to $2373.8 \mathrm{~m}$. In the Himalayas, monsoon winds are the principal source of precipitation as well as the primary governing factor of erosion, topography, environment, and vegetation [31]. The valley's vegetation features Sino-Japanese characteristics [32,33]. Summers are chilly and dry, and the valley has a dry-temperate climate with considerable seasonal variations. Between November and April, there is a lot of snow (an average annual snowfall of $3 \mathrm{~m}$ ). With rising altitude, the range represents a significant spike in snow depth. In comparison to lower elevations, the winter is severe at higher altitudes [34]. From January to April, there is a distinct wet season, with June to November being the driest months of the year.

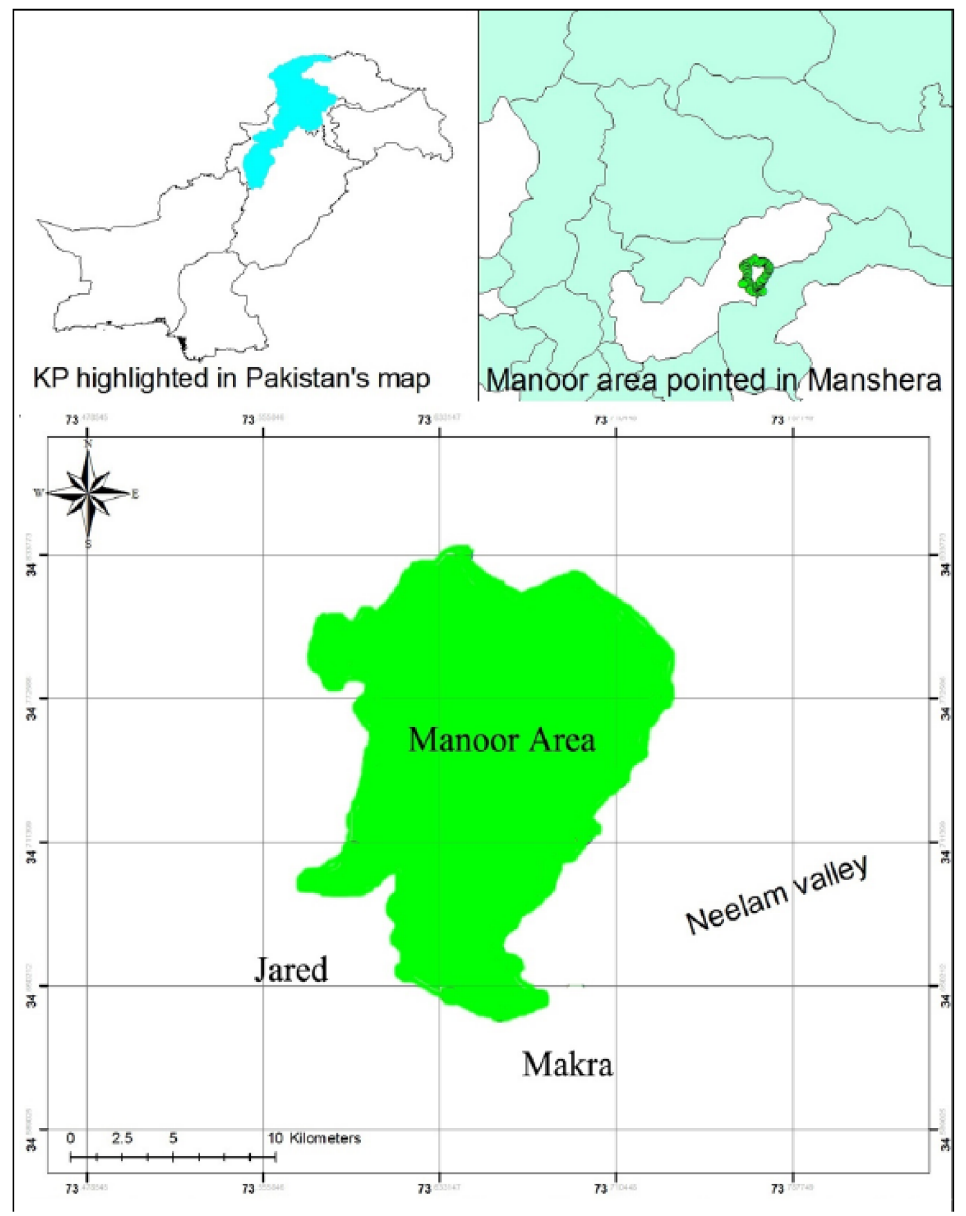

Figure 1. Map of the study area (Manoor Valley/Area) generated by ArcGIS software. 


\subsection{Vegetation Sampling and Plant Identification}

The vegetation of pure Deodar forests was surveyed and quantified [35] along the geographic, slope, edaphic, and climatic variables [36]. A line transect ecological technique was used for the vegetation sampling [35,37-41]. Sampling was carried out during 2015-2018. The surveyed sites were divided into 23 stands, and three sampling points within each stand were sampled along a 50 meter transect (total $=69$ transects). The phytosociological attributes (i.e., density, frequency, cover and their relative values, and importance value) were used for the calculation of the data from each sampled point [42-44]. The IV was further used to rank each species, and those with the highest IV were considered as the dominant species [42,43]. Similarly, plant communities were named after three dominant species [45-49]. The slope angle, aspect (i.e., east, west, south, and north), and exposure of each sampled point were recorded using a clinometer, and altitude, longitude, and latitude were measured by the geographical positioning system (GPS). Plant collection, labeling, pressing, and other herbarium work methodologies were adopted [50-56]. Their identification was confirmed using Flora of Pakistan [57-59], and they were submitted to the Hazara University herbarium in Mansehra (Pakistan).

\subsection{Environmental Gradients}

Soil samples weighing 200-400 g were collected from three randomly selected transects $(0-30 \mathrm{~cm}$ depth) within the studied vegetation area $[60,61]$. The replicated samples collected from each stand were thoroughly mixed to make a composite sample [62], stored in a sterile polythene bag, and labeled with a permanent marker. The samples were cleaned of any raw materials, such as rocks and stones, and then dried in the shade. Physicochemical tests were performed, including soil texture (clay, sand, silt, and loam), $\mathrm{pH}$ [63], electrical conductivity (EC) [64], organic matter $(\mathrm{OM})[65]$, nitrogen $(\mathrm{N})$, phosphorus $(\mathrm{P})$, potassium $(\mathrm{K})$, and calcium carbonate (CaCO3) contents [66]. Other climatic variables (such as temperature, barometric pressure (BP), wet bulb (WB), dew point (DP), heat index (HI), wind speed(WS), and humidity) were measured by recording data at each transect with a small remote weather station (Kestrel weather tracker 4000), and then calculating average values at the stand level [36].

\subsection{Statistical Analyses}

The data from all of the plant species, geographic, and other environmental gradients were compiled to see whether there was a link between them $[67,68]$. Various software packages, such as PC-ORD [69] and R 3.6.1 [70], were applied to analyze IV matrices (abundance of the 162 species) and values of geographic and environmental gradients obtained at 23 stands. PC-ORD software [35] was used to identify plant communities [36] by processing cluster analysis (CA), two-way cluster analysis (TWCA) [71], and two-way indicator species analysis (TWINSPAN). TWINSPAN was processed for the identification of the plant communities [35] based on species classification and sample clustering [72]. RStudio 4.0.0 [70] was used for processing non-multidimensional scaling ordination (NMDS) and principal component analysis (PCA) [73], ternary plot using the "Ternary" package [74], GLM with Gaussian error distribution and Likelihood ratio test [75], the spatial turnover and nestedness-resultant components of beta diversity, dissimilarity analysis [76,77], canonical correspondence analysis (CCA), and variation partitioning tests (partial CCA) [70]. The NMDS and PCA bi-plot ordinations were used to evaluate the correlation of environmental gradients with communities. The ternary diagram (simplex plots/Gibbs triangles) was drawn using the standard graphics functions by employing the soil texture variables data (sand, silt, and clay). GLM was conducted to compare the variables (20 variables distributed into four different groups: geographic, edaphic, climatic, and slope) evaluated among the communities produced by our previous analysis [75]. For calculating species richness by using a Poisson error distribution, we used a GLM, followed by a Likelihood ratio test. In addition, we used Gaussian error to measure each plant community's evenness, Shannon, and Simpson diversity. In the case of CCA and partial CCA, analyses fitted a complete 
model, and by using the step function with permutation in the package "stats", we reduced this complete model to the best model with the lowest number of variables. In addition, we used the Variance Inflation Factor (VIF) to validate the model's applicability by evaluating multicollinearity between variables in the final model.

\section{Results}

In this phytosociological investigation, 162 plant species were documented from 23 stands sampled in the pure Deodar forests of the Manoor Valley, Himalaya, Pakistan (Table 1). The pure Deodar forests ranged from 1580.8 to $2373.8 \mathrm{~m}$.

Table 1. List of plant species, with their importance values (IVs) calculated based on vegetative characteristics of each stand. Plant communities were named based upon three dominant species. The indicator species' IVs are mentioned in bold, as well, with respect to plant community.

\begin{tabular}{|c|c|c|c|}
\hline \multirow[b]{2}{*}{ Plant Species } & \multicolumn{3}{|c|}{ Plant Communities } \\
\hline & $\begin{array}{l}\text { Cedrus-Isodon- } \\
\text { Cynodon }\end{array}$ & $\begin{array}{l}\text { Cedrus-Cynodon- } \\
\text { Dryopteris }\end{array}$ & $\begin{array}{l}\text { Sambucus-Cedrus- } \\
\text { Desmodium }\end{array}$ \\
\hline Abies pindrow (Royle ex D. Don) Royle & 0.00 & 0.00 & 1.10 \\
\hline Achyranthes aspera $\mathrm{L}$. & 0.34 & 1.09 & 0.00 \\
\hline Achyranthes bidentata Blume & 0.21 & 0.20 & 0.00 \\
\hline Adiantum capillus-veneris L. & 3.67 & 3.86 & 1.45 \\
\hline Adiantum indicum J. Ghatak & 0.00 & 0.00 & 0.29 \\
\hline Adiantum venustum $\mathrm{D}$. Don & 0.00 & 0.00 & 0.35 \\
\hline Aesculus indica (Wall. ex Camb.) Hook. & 0.00 & 0.00 & 0.26 \\
\hline Ailanthus altissima (Mill.) Swingle & 0.00 & 3.62 & 0.80 \\
\hline Ajuga integrifolia Buch.-Ham. & 1.25 & 2.58 & 0.00 \\
\hline Amaranthus viridis L. & 0.00 & 1.37 & 0.00 \\
\hline Anagallis arvensis $\mathrm{L}$. & 1.77 & 2.55 & 0.00 \\
\hline Arisaema flavum (Forssk.) Schott & 3.88 & 1.07 & 0.00 \\
\hline Artemisia absinthium L. & 0.00 & 5.04 & 1.39 \\
\hline Bergenia ciliata (Haw.) Sternb. & 0.00 & 0.00 & 0.56 \\
\hline Berberis lycium Royle & 5.03 & 0.00 & 0.00 \\
\hline Berberis parkeriana C.K. Schneid. & 0.00 & 0.00 & 0.61 \\
\hline Berberis pachyacantha Bien. ex Koehne & 0.00 & 0.00 & 0.20 \\
\hline Bistorta amplexicaulis (D. Don) Greene & 0.00 & 0.00 & 1.82 \\
\hline Bromus diandrus Roth. & 0.00 & 0.00 & 0.53 \\
\hline Bromus secalinus L. & 0.00 & 0.00 & 1.56 \\
\hline Bromus tectorum $\mathrm{L}$. & 0.00 & 0.00 & 0.68 \\
\hline Bupleurum nigrescens E. Nasir & 2.96 & 0.00 & 1.55 \\
\hline Calamintha umbrosa (M. Bieb.) Hedge & 0.00 & 0.00 & 1.42 \\
\hline Cannabis sativa $\mathrm{L}$. & 0.00 & 0.95 & 0.00 \\
\hline Capsella bursa-pastoris (L.) Medik. & 0.00 & 3.76 & 0.00 \\
\hline Cedrus deodara (Roxb. ex Lamb.) G. Don & 13.74 & 10.79 & 5.05 \\
\hline
\end{tabular}


Table 1. Cont.

\begin{tabular}{|c|c|c|c|}
\hline \multirow[b]{2}{*}{ Plant Species } & \multicolumn{3}{|c|}{ Plant Communities } \\
\hline & $\begin{array}{l}\text { Cedrus-Isodon- } \\
\text { Cynodon }\end{array}$ & $\begin{array}{l}\text { Cedrus-Cynodon- } \\
\text { Dryopteris }\end{array}$ & $\begin{array}{l}\text { Sambucus-Cedrus- } \\
\text { Desmodium }\end{array}$ \\
\hline Cichorium intybus L. & 0.00 & 0.46 & 0.00 \\
\hline Cirsium arvense (L.) Scop. & 2.00 & 3.18 & 0.21 \\
\hline Clematis grata Wall. & 2.46 & 3.97 & 0.64 \\
\hline Clinopodium vulgare $\mathrm{L}$. & 0.00 & 2.53 & 0.51 \\
\hline Commelina benghalensis L. & 0.00 & 0.00 & 0.26 \\
\hline Convolvulus arvensis $\mathrm{L}$. & 4.41 & 2.26 & 0.00 \\
\hline Conyza japonica (Thunb.) Less. ex Less. & 0.00 & 0.39 & 0.23 \\
\hline Corydalis carinata Lidén and Z.Y.Su & 0.00 & 0.00 & 0.03 \\
\hline Corydalis virginea Lidén and Z.Y.Su & 0.00 & 0.00 & 0.05 \\
\hline Cotoneaster acuminatus Wall. ex Lindl. & 0.00 & 0.00 & 0.16 \\
\hline Cotoneaster microphyllus Wall. ex Lindl & 3.71 & 0.00 & 0.00 \\
\hline Crotalaria sp. & 0.00 & 0.67 & 0.22 \\
\hline Cuscuta reflexa Roxb. & 1.63 & 0.00 & 0.00 \\
\hline Cynodon dactylon (L.) Pers. & 10.47 & 10.40 & 1.83 \\
\hline Cynoglossum glochidiatum Wall. ex Benth. & 1.19 & 1.90 & 0.00 \\
\hline Cyperus odoratus L. & 0.00 & 1.58 & 0.78 \\
\hline Cyperus rotundus L. & 5.48 & 3.26 & 1.66 \\
\hline Dactylis glomerata $\mathrm{L}$. & 0.00 & 0.00 & 3.30 \\
\hline Desmodium elegans DC. & 0.00 & 0.00 & 4.10 \\
\hline Dicliptera bupleuroides Nees & 0.00 & 0.49 & 0.10 \\
\hline Dioscorea deltoidea Wall. ex Griseb. & 0.00 & 0.00 & 0.04 \\
\hline Diospyros lotus L. & 0.00 & 0.83 & 0.13 \\
\hline Dipsacus inermis Wall. in Roxb. & 0.00 & 0.00 & 0.06 \\
\hline Dodonaea viscosa (L.) Jacq. & 0.30 & 0.54 & 0.00 \\
\hline Dryopteris wallichiana (Spreng.) Hyl. & 5.88 & 5.48 & 3.26 \\
\hline Duchesnea indica (Andx) Fake. & 4.07 & 1.06 & 0.61 \\
\hline Erigeron canadensis L. & 0.00 & 0.00 & 0.50 \\
\hline Euphrasia himalayica Wetts. & 0.00 & 0.00 & 1.50 \\
\hline Euphorbia hirta L. & 0.00 & 1.72 & 0.00 \\
\hline Euphorbia prostrata Ait. & 0.00 & 0.70 & 0.00 \\
\hline Filipendula vestita (Wall. ex G. Don.) Maxim. & 0.00 & 0.00 & 0.70 \\
\hline Foeniculum vulgare Mill. & 0.00 & 0.00 & 2.38 \\
\hline Fragaria nubicola (Hook. f.) Lindl. ex Lacaita & 6.41 & 2.64 & 2.45 \\
\hline Fumaria indica (Hausskn) Pugsley & 1.29 & 0.00 & 0.00 \\
\hline Galium aparine $\mathrm{L}$ & 0.00 & 0.00 & 0.48 \\
\hline Geranium nepalense Sweet. & 0.00 & 0.41 & 0.49 \\
\hline Geranium wallichianum D. Don ex Sweet & 4.00 & 1.20 & 1.19 \\
\hline Grewia optiva J.R.Drumm. ex Burret & 0.00 & 0.00 & 0.16 \\
\hline
\end{tabular}


Table 1. Cont.

\begin{tabular}{|c|c|c|c|}
\hline \multirow[b]{2}{*}{ Plant Species } & \multicolumn{3}{|c|}{ Plant Communities } \\
\hline & $\begin{array}{l}\text { Cedrus-Isodon- } \\
\text { Cynodon }\end{array}$ & $\begin{array}{l}\text { Cedrus-Cynodon- } \\
\text { Dryopteris }\end{array}$ & $\begin{array}{l}\text { Sambucus-Cedrus- } \\
\text { Desmodium }\end{array}$ \\
\hline Hedera nepalensis K. Koch & 4.25 & 1.96 & 0.00 \\
\hline Heracleum candicans Wall. ex DC. & 0.00 & 0.00 & 3.29 \\
\hline Hyoscyamus niger L. & 0.00 & 0.00 & 0.47 \\
\hline Impatiens bicolor Royle. & 5.64 & 2.41 & 0.94 \\
\hline Indigofera hebepetala Baker & 0.00 & 0.00 & 0.91 \\
\hline Indigofera heterantha Brandis & 0.00 & 3.68 & 1.52 \\
\hline Ipomoea nil (L.) Roth & 0.00 & 1.67 & 0.64 \\
\hline Isodon rugosus (Wall. ex Benth.) Codd & 11.50 & 3.28 & 0.24 \\
\hline Juglans regia L. & 0.00 & 1.17 & 0.08 \\
\hline Justicia adhatoda L. & 0.00 & 0.00 & 0.29 \\
\hline Lamium amplexicaule $\mathrm{L}$. & 0.00 & 0.98 & 0.00 \\
\hline Lathyrus aphaca L. & 0.00 & 0.00 & 0.53 \\
\hline Launaea procumbens (Roxb.) Ramayya and Rajagopal & 1.11 & 1.49 & 0.08 \\
\hline Leptodermis virgata Edgew. ex Hook.F. & 3.16 & 2.99 & 0.00 \\
\hline Lindelofia sp. & 0.74 & 0.46 & 0.02 \\
\hline Malvastrum coromandelianum(L.) Garcke & 0.28 & 0.25 & 0.09 \\
\hline Malva parviflora $\mathrm{L}$ & 0.00 & 2.46 & 0.00 \\
\hline Malva neglecta Wallr. & 0.00 & 2.86 & 0.00 \\
\hline Medicago sativa $\mathrm{L}$ & 4.95 & 3.83 & 0.55 \\
\hline Micromeria biflora (Ham.) Bth. & 0.00 & 2.64 & 0.00 \\
\hline Nepeta graciliflora Benth. & 0.00 & 0.00 & 0.46 \\
\hline Nepeta laevigata (D. Don) Hand.-Mazz & 0.00 & 0.00 & 0.97 \\
\hline Oenothera rosea L. Her ex Aiton & 0.00 & 0.99 & 0.30 \\
\hline Olea ferruginea Wall. ex Aitch. & 0.00 & 0.00 & 0.12 \\
\hline Onopordum acanthium L. & 0.00 & 0.00 & 2.86 \\
\hline Origanum majorana L. & 0.00 & 0.00 & 0.16 \\
\hline Origanum vulgare L. & 0.00 & 0.00 & 1.54 \\
\hline Oxalis corniculata $\mathrm{L}$. & 4.11 & 5.38 & 0.81 \\
\hline Parthenium hysterophorus L. & 0.00 & 0.91 & 0.00 \\
\hline Parrotiopsis jacquemontiana (Decne.) Rehder & 6.41 & 0.81 & 2.37 \\
\hline Paspalum dilatatun Poir. & 0.00 & 0.00 & 0.28 \\
\hline Pennisetum orientale Rich. & 0.00 & 0.00 & 3.04 \\
\hline Periploca aphylla Decne. & 0.00 & 0.00 & 0.20 \\
\hline Persicaria capitata (Buch.-Ham. ex D.Don) H.Gross & 2.62 & 2.86 & 0.00 \\
\hline Phragmites altissimus (Benth.) Mabille & 0.00 & 0.00 & 1.88 \\
\hline Phytolacca americana $\mathrm{L}$. & 0.00 & 0.00 & 0.08 \\
\hline Phytolacca latbenia (Moq.) H. Walter & 0.00 & 0.00 & 0.04 \\
\hline Pimpinella stewartii (Dunn) Nasir & 0.00 & 2.10 & 1.53 \\
\hline Pinus roxburghii Sarg & 0.43 & 0.10 & 0.00 \\
\hline
\end{tabular}


Table 1. Cont.

\begin{tabular}{|c|c|c|c|}
\hline \multirow[b]{2}{*}{ Plant Species } & \multicolumn{3}{|c|}{ Plant Communities } \\
\hline & $\begin{array}{l}\text { Cedrus-Isodon- } \\
\text { Cynodon }\end{array}$ & $\begin{array}{l}\text { Cedrus-Cynodon- } \\
\text { Dryopteris }\end{array}$ & $\begin{array}{l}\text { Sambucus-Cedrus- } \\
\text { Desmodium }\end{array}$ \\
\hline Pinus wallichiana A.B. Jacks & 0.00 & 0.62 & 0.77 \\
\hline Piptatherum aequiglume (Duthie ex Hook.f.) Roshev. & 0.00 & 0.00 & 0.68 \\
\hline Plantago major L. & 0.00 & 0.00 & 0.33 \\
\hline $\begin{array}{l}\text { Pleurospermum stellatum (D. Don) Benth. ex C.B. } \\
\text { Clarke }\end{array}$ & 0.00 & 0.00 & 0.04 \\
\hline Pleurospermum stylosum C.B. Clarke & 0.00 & 0.00 & 0.04 \\
\hline Poa infirma Kunth & 0.00 & 0.00 & 1.72 \\
\hline Polygonum plebeium R.Br. & 0.00 & 0.00 & 0.91 \\
\hline Polygonatum sp. & 0.00 & 0.00 & 0.03 \\
\hline Polygonatum verticillatum (L.) Allioni & 0.00 & 0.00 & 0.25 \\
\hline Populus alba L. & 0.00 & 0.00 & 1.80 \\
\hline Populus ciliata Wall. ex Royle & 0.00 & 0.00 & 0.33 \\
\hline Populus nigra L. & 0.00 & 0.00 & 0.16 \\
\hline Portulaca oleracea L. & 0.00 & 0.00 & 0.28 \\
\hline Potentilla nepalensis Hook. & 0.00 & 0.00 & 0.36 \\
\hline Prunella vulgaris $\mathrm{L}$. & 0.00 & 0.00 & 1.62 \\
\hline Pteridium aquilinum (L.) Kuhn & 0.00 & 0.46 & 0.00 \\
\hline Pteracanthus urticifolius (Wall. ex Kuntze) Bremek. & 0.00 & 0.00 & 0.07 \\
\hline Ranunculus muricatus L. & 0.00 & 0.00 & 0.44 \\
\hline Reinwardtia trigyna Planch. & 0.00 & 0.00 & 0.26 \\
\hline Rhamnus purpurea Edgew. & 0.00 & 0.00 & 0.14 \\
\hline Rhynchosia pseudo-cajan Cambess. & 0.00 & 0.00 & 0.13 \\
\hline Robinia pseudo-accacia L. & 0.00 & 0.00 & 0.22 \\
\hline Rosa brunonii Lindl. & 0.00 & 0.00 & 0.80 \\
\hline Rubus fruticosus agg. & 0.00 & 0.00 & 1.04 \\
\hline Rubus sanctus Schreber & 0.00 & 0.00 & 0.34 \\
\hline Rydingia limbata (Benth.) Scheen and V.A. Albert & 0.00 & 0.00 & 0.26 \\
\hline Saccharum spontaneum L. & 0.00 & 0.00 & 0.06 \\
\hline Salix alba $\mathrm{L}$ & 0.00 & 0.00 & 0.25 \\
\hline Salix denticulata subsp. hazarica (R. Parker) Ali & 0.00 & 0.00 & 0.08 \\
\hline Salvia lanata Roxb. & 0.00 & 0.79 & 0.39 \\
\hline Salvia moorcroftiana Wall. ex Benth. & 0.94 & 0.00 & 0.00 \\
\hline Salvia nubicola Wall. ex Sweet & 0.00 & 0.00 & 0.61 \\
\hline Salix tetrasperma Roxb & 0.00 & 0.00 & 0.03 \\
\hline Sambucus wightiana Wall. ex Wight and Arn & 0.00 & 0.00 & 5.25 \\
\hline Saussurea sp. & 0.00 & 0.35 & 0.00 \\
\hline Seseli libanotis (L.) W.D.J. Koch & 0.00 & 0.00 & 0.04 \\
\hline Sida cordata (Burm.f.) Borss & 0.00 & 0.00 & 0.03 \\
\hline Silene conoidea $\mathrm{L}$. & 0.00 & 0.00 & 0.42 \\
\hline
\end{tabular}


Table 1. Cont.

\begin{tabular}{|c|c|c|c|}
\hline \multirow[b]{2}{*}{ Plant Species } & \multicolumn{3}{|c|}{ Plant Communities } \\
\hline & $\begin{array}{l}\text { Cedrus-Isodon- } \\
\text { Cynodon }\end{array}$ & $\begin{array}{l}\text { Cedrus-Cynodon- } \\
\text { Dryopteris }\end{array}$ & $\begin{array}{l}\text { Sambucus-Cedrus- } \\
\text { Desmodium }\end{array}$ \\
\hline Silybum marianum (L.) Gaertn. & 0.00 & 0.61 & 0.00 \\
\hline Silene vulgaris (Moench) Garcke & 0.00 & 0.00 & 0.62 \\
\hline Smilax glaucophylla Koltzsch & 0.00 & 0.00 & 0.20 \\
\hline Sonchus asper (L.) Hill & 0.00 & 0.95 & 0.49 \\
\hline Sorghum halepense (L.) Pers. & 3.37 & 1.91 & 1.17 \\
\hline Sorbaria tomentosa (Lindl.) Rehder & 0.00 & 1.00 & 3.62 \\
\hline Spiraea affinis R.Parker & 0.00 & 0.00 & 0.23 \\
\hline Spiraea vaccinifolia D. Don & 0.00 & 0.00 & 0.02 \\
\hline Sporobolus diandrus (Retz.) P.Beauv. & 0.00 & 0.00 & 0.70 \\
\hline Stellaria media (L.) Vill. & 0.00 & 0.00 & 0.12 \\
\hline Tagetes minuta $\mathrm{L}$. & 0.00 & 4.33 & 0.77 \\
\hline Taraxacum officinale aggr. F.H. Wigg. & 0.00 & 1.52 & 0.00 \\
\hline Thalictrum pedunculatum Edgew. & 0.00 & 0.00 & 0.03 \\
\hline Torilis japonica (Houtt.) DC. & 0.00 & 0.00 & 0.04 \\
\hline Trachyspermum amii (L.) Sprague & 0.00 & 0.00 & 0.01 \\
\hline Trifolium repens L. & 3.26 & 3.60 & 0.00 \\
\hline Vicia sativa $\mathrm{L}$. & 0.00 & 0.00 & 0.04 \\
\hline Vincetoxicum petrense (Hemsl. and Lace) Rech. f. & 0.00 & 0.00 & 0.04 \\
\hline Viola odorata L. & 0.00 & 0.00 & 0.29 \\
\hline Viola serpens Wall. Ex Ging & 0.00 & 0.00 & 0.36 \\
\hline Vitex negundo L. & 0.00 & 0.00 & 0.19 \\
\hline
\end{tabular}

\subsection{Cluster Analysis (CA), Two-Way Cluster Analysis (TWCA) and TWINSPAN}

Cluster analysis (CA), using PC-ORD, grouped the vegetation into three plant communities, as indicated in the dendrograms under the impact of various environmental gradients (Figure 2). TWCA displays the detailed distribution patterns of 162 plant species recorded at 23 sampling sites (stands). Based on influenced environmental gradients, all of these stands were clustered into three distinct groups (plant communities) (Figure 3). According to TWINSPAN classification, a total of three communities (Cedrus-Isodon-Cynodon, Cedrus-Cynodon-Dryopteris, and Sambucus-Cedrus-Desmodium) were established by clustering 162 reported plants species in 23 stands under the strong influence of environmental variables in pure Deodar forests (Table 1). 


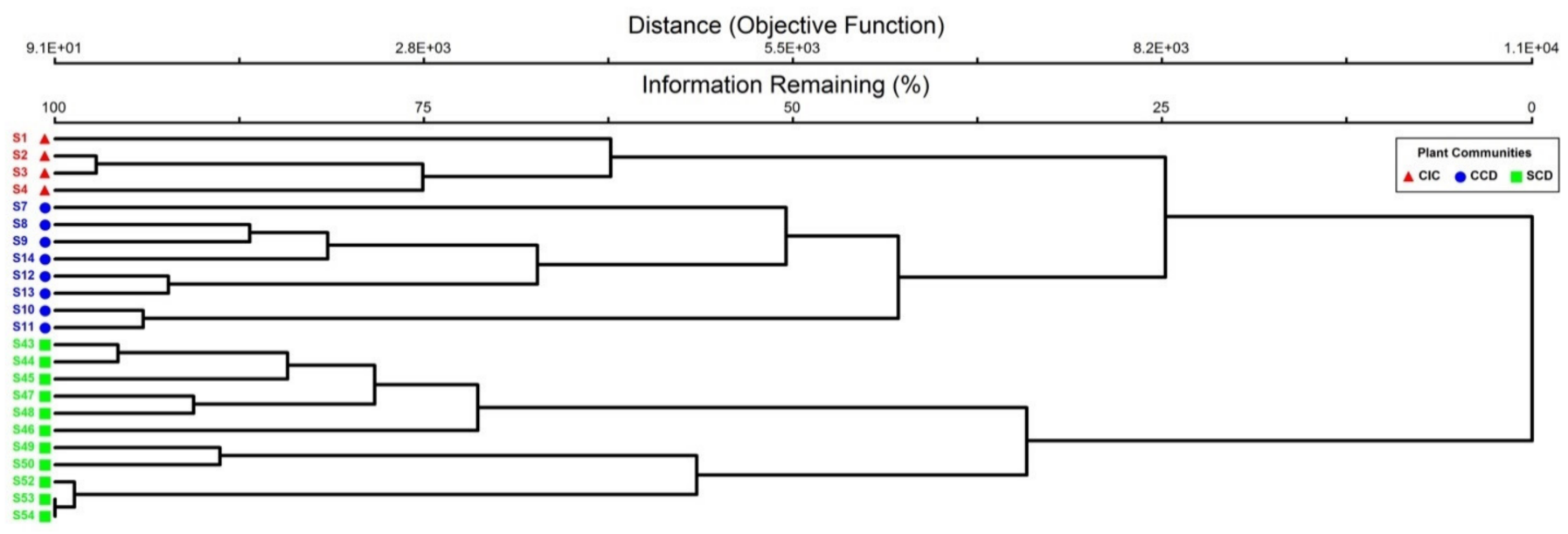

Figure 2. Cluster analysis displaying three different plant communities' recognition based upon the grouping of 23 stands and 162 plant species. CIC, Cedrus-Isodon-Cynodon; CCD, Cedrus-CynodonDryopteris; SCD, Sambucus-Cedrus-Desmodium.

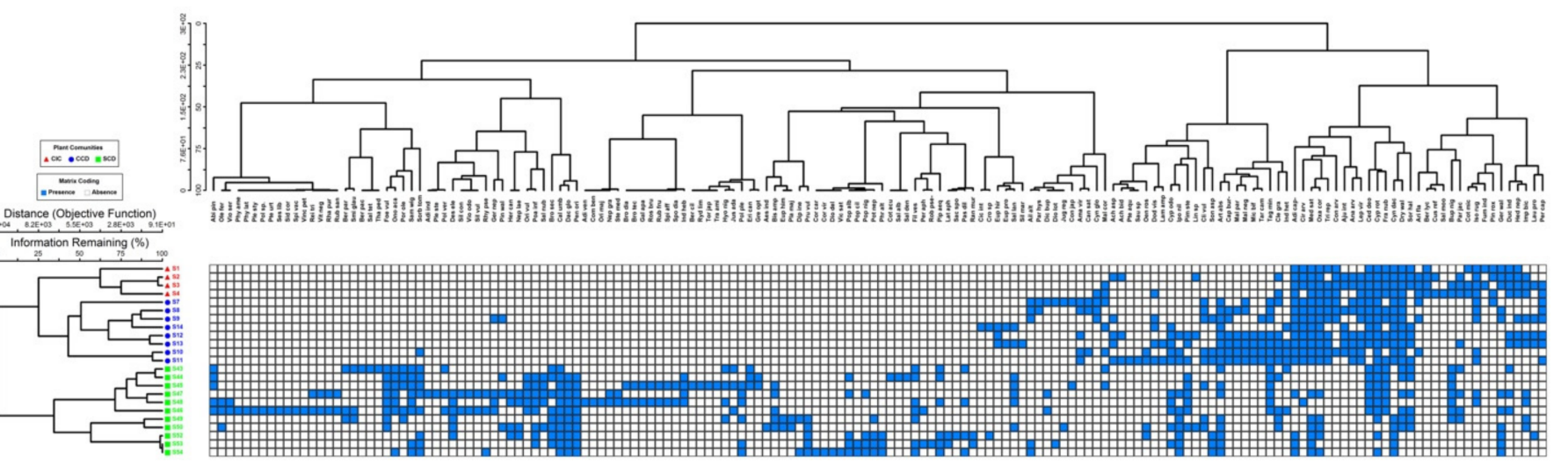

Figure 3. TWCA analysis displaying three distinct communities recognized based on 162 plant species recorded from 23 stands. Each blue dot represents the existence of a species, whereas each white dot indicates its absence. (For the complete names of plant communities, see Figure 2).

\subsection{Community Distribution Modeling along the Environmental Gradients}

NMDS (Figure 4a-d) and PCA are used to show the relationship between identified communities and environmental gradients (Figure 4e). The most representative environmental factors that drove the plant community structure were altitude, slope angle and aspects, NE, slope SW, K, pH, OM, loam, silt, sand, clay, temperature, HI, and WS (Table 2). Environmental gradients distinguish the 23 sample sites into 3 communities in both ordinations, as TWINSPAN, CA (Figure 2), and TWCA have already revealed (Figure 3). 


\section{a) Geographic}

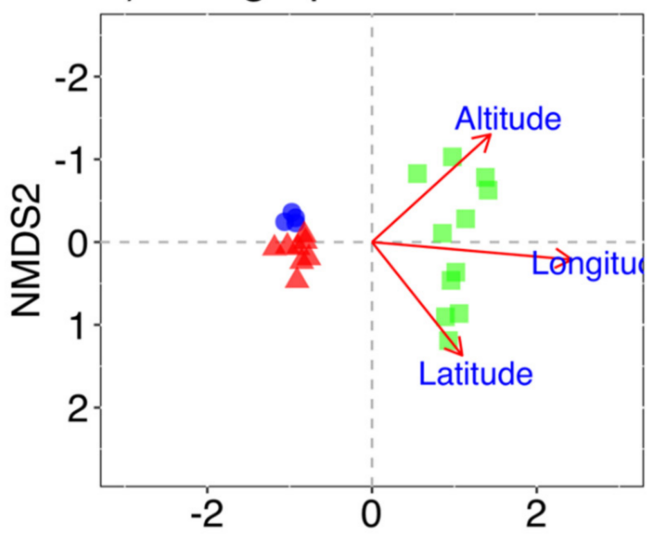

c) Edaphic

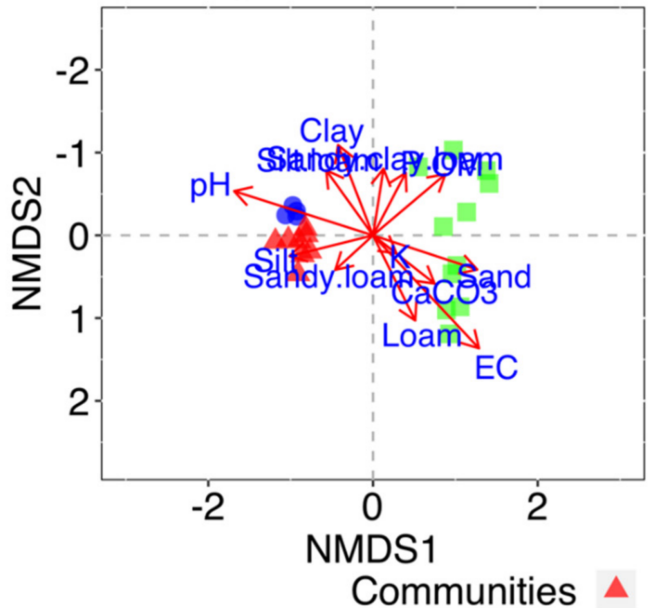

b) Slope

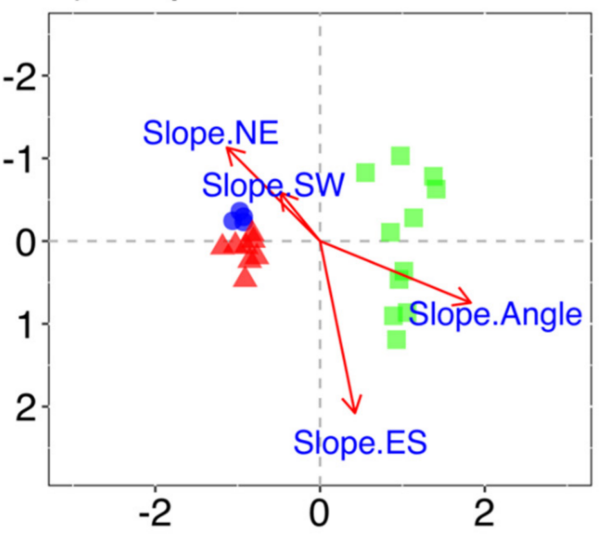

d) Climatic

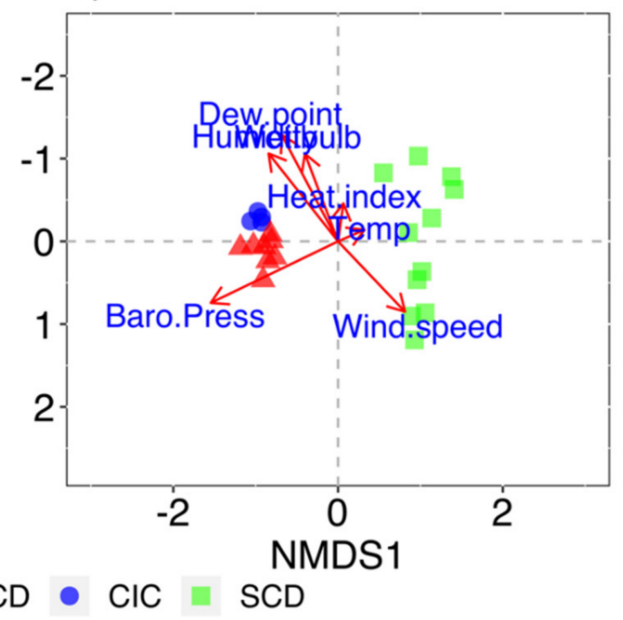

e) PCA

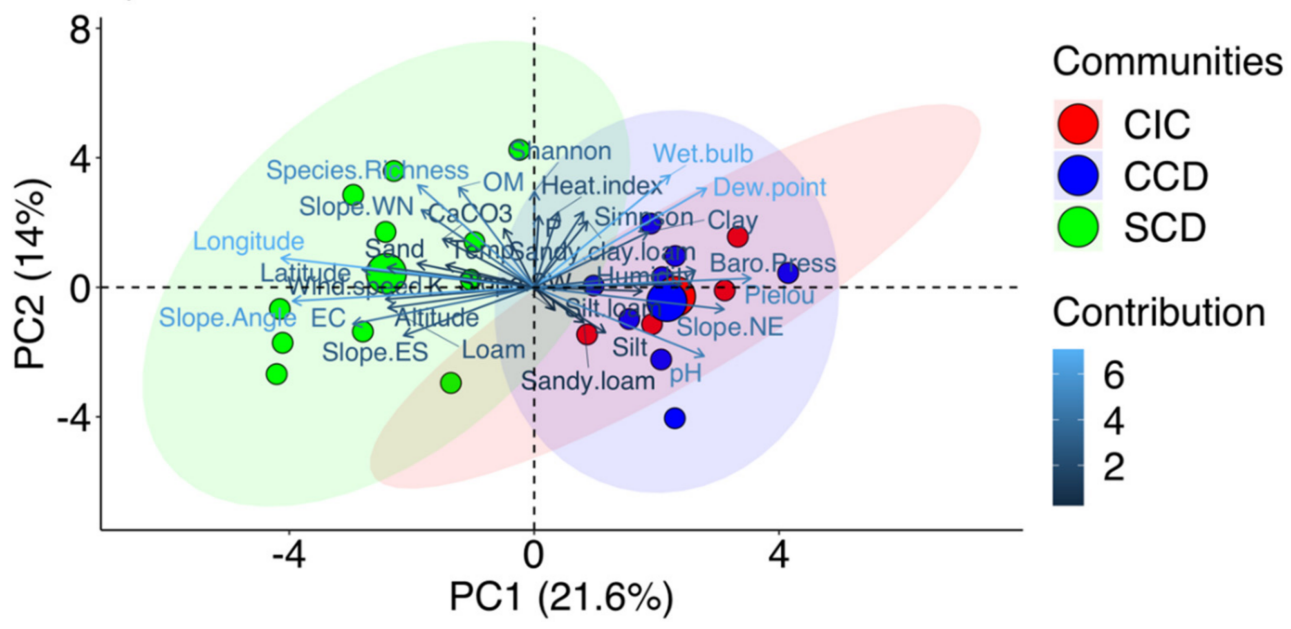

Figure 4. Multivariate analyses among plant communities of pure Deodar forest and environmental variables. (a-d) Non-Multidimensional Scaling (NMDS) of three plant communities and their ecological variables such as geographic (a), slope (b), edaphic (c), and climatic (d). (e) Principal Component Analysis (PCA) ordination of three communities along the environmental variables. For the complete names of plant communities, see Figure 2. 
Table 2. Means of geographic and environmental gradients measured at three different transects of each stand were recorded (wind speed averages are given as integers).

\begin{tabular}{|c|c|c|c|c|c|c|c|c|c|c|c|c|c|c|c|c|c|c|c|c|}
\hline Stands & Altitude & Latitude & Longitude & SA & Temp & $\mathbf{H}$ & HI & WS & DP & WB & BP & $\mathrm{pH}$ & EC & OM & $\mathrm{CaCO}_{3}$ & $\mathbf{K}$ & $\mathbf{P}$ & Sand & Silt & Clay \\
\hline S1 & 1807.9 & 34.69282 & 73.58045 & 45 & 23.7 & 68.9 & 24.4 & 1 & 17.6 & 19.4 & 826.1 & 6.4 & 0.56 & 1.1 & 9.2 & 210 & 17 & 21.4 & 54 & 24.6 \\
\hline S2 & 1769.8 & 34.69304 & 73.58070 & 38 & 23.3 & 72.5 & 25 & 1 & 19.1 & 20.2 & 877.5 & 6 & 1.54 & 1.07 & 7.7 & 216 & 13 & 45.8 & 28 & 26.2 \\
\hline S3 & 1792.4 & 34.69279 & 73.58091 & 34 & 27.1 & 55.2 & 29 & 1 & 17.3 & 19.7 & 815.1 & 6.1 & 1.5 & 0.59 & 9.6 & 210 & 13 & 15.6 & 58.2 & 26.2 \\
\hline S4 & 1869.5 & 34.69168 & 73.58167 & 40 & 26.4 & 57 & 28.8 & 2 & 17 & 19.5 & 822.1 & 6.7 & 2.24 & 0.7 & 3.4 & 215 & 6.1 & 46 & 31 & 23 \\
\hline S5 & 1580.8 & 34.69881 & 73.60361 & 35 & 26.9 & 60.2 & 28.4 & 1 & 19.4 & 21.3 & 836.7 & 6.2 & 0.73 & 1.07 & 9.7 & 235 & 16 & 26.4 & 49.2 & 24.4 \\
\hline S6 & 1587.5 & 34.69675 & 73.57915 & 24 & 27.8 & 56.6 & 28.8 & 1 & 18.5 & 21 & 836 & 6.1 & 1.13 & 0.6 & 9.5 & 200 & 11 & 15.8 & 58 & 26.2 \\
\hline S7 & 1598.2 & 34.69641 & 73.58026 & 28 & 28.4 & 53.9 & 29.2 & 0 & 18.1 & 20.8 & 834.9 & 6.5 & 1.92 & 1.15 & 7.4 & 205 & 11 & 35.8 & 50 & 14.2 \\
\hline S8 & 1872.9 & 34.68000 & 73.58000 & 35 & 25 & 56.2 & 26.3 & 1 & 15.5 & 18.4 & 807.1 & 6.7 & 1.49 & 0.63 & 2.8 & 210 & 10 & 55 & 26 & 19 \\
\hline S9 & 1864.6 & 34.68760 & 73.58897 & 50 & 25.8 & 53.6 & 26.5 & 0 & 17.2 & 19.7 & 807.7 & 5.9 & 1.6 & 1.25 & 5.9 & 215 & 12 & 29.4 & 56 & 14.6 \\
\hline $\mathrm{S} 10$ & 1968.3 & 34.68724 & 73.58803 & 36 & 25.4 & 60.3 & 26.8 & 0 & 18.9 & 20.7 & 797.6 & 6.7 & 0.93 & 1.08 & 2.8 & 225 & 11 & 42 & 32 & 26 \\
\hline S11 & 1965.5 & 34.68650 & 73.58700 & 40 & 20.9 & 74.9 & 22.1 & 1.5 & 16.8 & 18.2 & 797 & 6.4 & 1.73 & 0.85 & 5.7 & 205 & 15 & 43 & 35 & 22 \\
\hline S12 & 1982.0 & 34.68704 & 73.58770 & 45 & 21.8 & 69.2 & 22.4 & 0 & 16.6 & 18.2 & 796.3 & 7.3 & 0.84 & 0.64 & 6.6 & 215 & 7.7 & 29.6 & 44 & 26.4 \\
\hline $\mathrm{S} 13$ & 2373.8 & 34.69443 & 73.62392 & 85 & 26.3 & 53.5 & 26.9 & 0 & 16.2 & 18.6 & 758.5 & 6.9 & 2.31 & 0.65 & 2.7 & 215 & 8 & 29 & 57 & 14 \\
\hline S14 & 2352.1 & 34.69476 & 73.62369 & 60 & 27.3 & 59.1 & 28.4 & 0 & 18.2 & 20.6 & 760.6 & 5.7 & 1.8 & 1.19 & 7.5 & 211 & 16 & 48 & 30 & 22 \\
\hline $\mathrm{S} 16$ & 2080.2 & 34.69696 & 73.61943 & 85 & 25.9 & 59.5 & 26.2 & 1 & 17.2 & 19.4 & 786.6 & 5.4 & 1.43 & 1.1 & 11.8 & 225 & 11 & 42 & 32 & 26 \\
\hline $\mathrm{S} 17$ & 2013.7 & 34.69766 & 73.61852 & 75 & 26.9 & 57.3 & 27.9 & 2 & 17.6 & 19.9 & 793.2 & 5.6 & 1.95 & 1.2 & 7.5 & 212 & 13 & 46 & 29.6 & 24.4 \\
\hline $\mathrm{S} 18$ & 1986.3 & 34.69753 & 73.61784 & 25 & 26.3 & 54.1 & 30.7 & 1.5 & 18.5 & 21.1 & 795.7 & 6.2 & 2.48 & 1.2 & 8 & 206 & 15 & 48 & 31 & 21 \\
\hline $\mathrm{S} 19$ & 1949.4 & 34.69768 & 73.61755 & 85 & 25.6 & 53.9 & 25.6 & 3 & 16.1 & 18.8 & 799.3 & 5.7 & 3.71 & 1.2 & 10.3 & 228 & 14 & 33 & 48 & 19 \\
\hline S20 & 1943.6 & 34.69779 & 73.61757 & 75 & 26.4 & 57 & 26.6 & 2 & 16.9 & 19.9 & 800 & 5.6 & 2.39 & 1.1 & 11.9 & 225 & 15 & 40 & 36 & 24 \\
\hline $\mathrm{S} 21$ & 1936.0 & 34.69785 & 73.61739 & 65 & 25.6 & 47.9 & 26.3 & 1.5 & 13.7 & 17.4 & 800.7 & 5.5 & 9.18 & 0.6 & 13 & 216 & 11 & 41.8 & 42 & 16.2 \\
\hline $\mathrm{S} 22$ & 1942.4 & 34.71430 & 73.62436 & 70 & 25.5 & 49.9 & 26.2 & 2 & 15.2 & 18 & 800.2 & 6.5 & 2.02 & 0.86 & 5.5 & 215 & 16 & 44 & 36 & 20 \\
\hline S23 & 1947.6 & 34.71437 & 73.62418 & 70 & 25.4 & 52.9 & 25.4 & 1.5 & 16.2 & 18.3 & 799.5 & 5.2 & 3.99 & 1.06 & 7.4 & 210 & 5 & 47.8 & 36 & 16.2 \\
\hline
\end{tabular}


In constrained PCA ordination, the PC1 axis accounted for the most explanatory variance $(21.6 \%)$, whereas the PC2 axis accounted for the least (14\%). By distinguishing the vegetation of the pure Deodar forest into three communities (Figure $4 \mathrm{a}-\mathrm{e}$ ), the considerable effect of the altitudinal gradient was revealed. Altitude (1936-2373 m), slope angle $\left(25-85^{\circ}\right)$, and sandy $(29-48 \%)$ and loamy soil texture all indicated a positive and substantial association in the SCD community. Potassium $\left(216 \mathrm{mg} \mathrm{kg}^{-1}\right), \mathrm{EC}\left(3.3 \mathrm{dsm}^{-1}\right), \mathrm{CaCO}_{3}$ $\left(8.45 \mathrm{mg} \mathrm{kg}^{-1}\right)$, and organic matter $(1.03 \%)$ were the edaphic variables that shaped this plant community. Furthermore, wind speed $\left(1.45 \mathrm{~ms}^{-1}\right)$ and temperature $\left(25.8^{\circ} \mathrm{C}\right)$ were found to have a positive and significant association. In contrast with this, the CCD community was primarily found in the lower mountainous ranges (1580.8-1982 m.a.s.1.) and showed positive and significant relationships with the northeastern slope, silty (32-58\%) and sandy (15.8-55\%) loamy soil texture, and barometric pressure (814.3 pa). Nonetheless, the CIC community was recognized at the middle altitudinal range (1769.8-1869.5 m). This community revealed a significant positive correlation with the northeastern to southwestern slope, $\mathrm{pH}$ (6.3), wet bulb (19.7), and dew point (17.7). Since silt, sand, and clay were representative in both NMDS and PCA (Figure 4) and are expressed in percentage (Table 2), the relationship of CIC, CCD, and SCD plant communities was plotted with the said three edaphic elements in a ternary plot (Figure 5). Likewise, a clear clustering in plant communities was observed according to soil composition based on these three elements. Based on the mean values, the CCD and CIC communities were hosted by soils having the maximum silty texture, i.e., $43.7 \%$ and $42.8 \%$, respectively. On the other hand, the SCD community was hosted on soil that comprised a higher sandy texture $(41.7 \%)$. As each plant community comprised several stands, the soil-texture values recorded for each stand were found to have no significant differences (Figure 5). These results reveal that the tree layer determines the structure of the ground cover more than soil does.

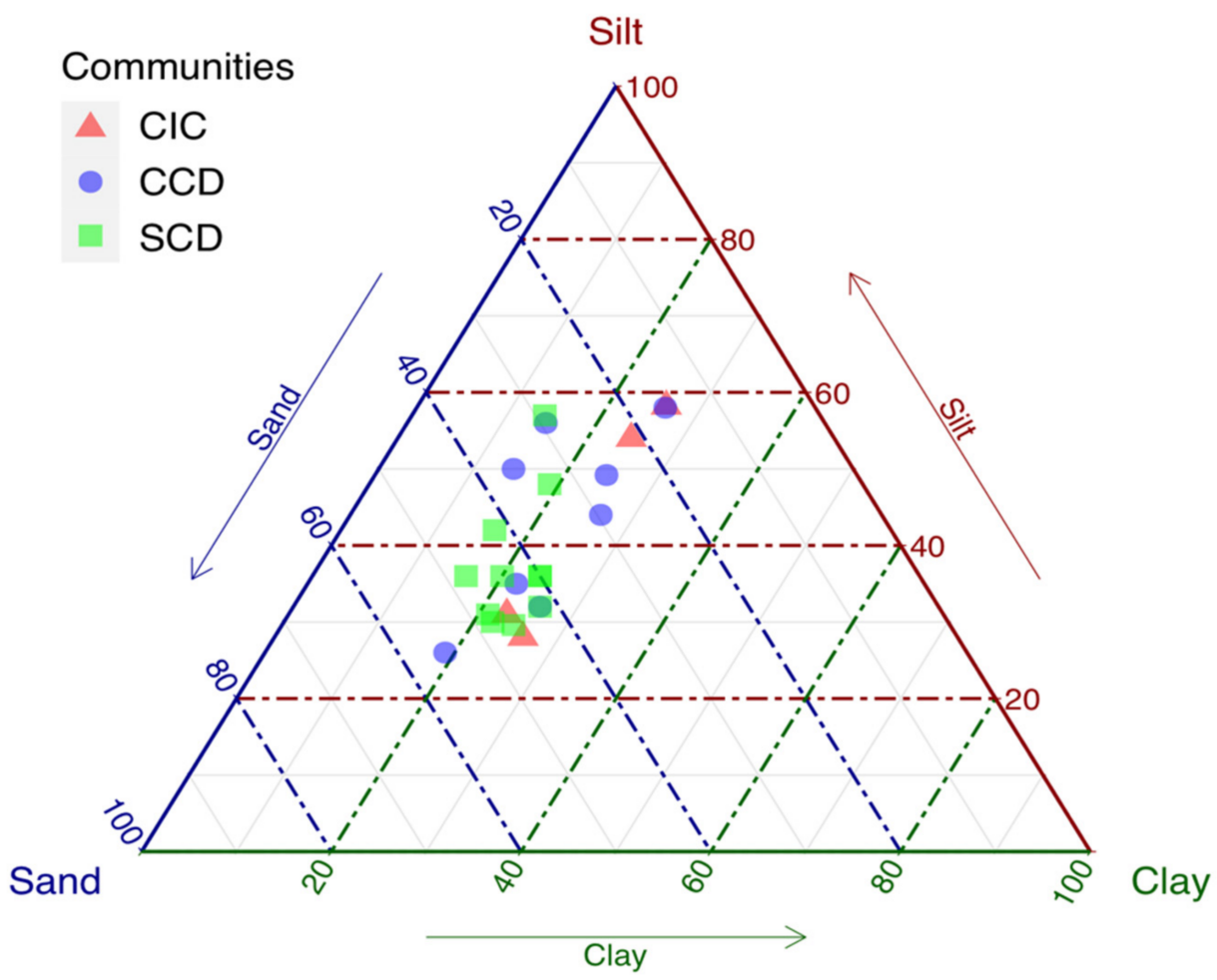

Figure 5. Ternary plot demonstrating the distribution pattern of communities on the basis of soil texture (clay, sand, and silt). (For the complete names of plant communities, see Figure 2).

\subsection{Significance Testing of Pure Deodar Forest Communities in Relation to Studied Variables}

The significance testing of recognized plant communities was performed in association with geographic and environmental gradients. The results of the GLM analyses revealed 
that the majority of the gradients were significantly different ( $p$-value $<0.05$; Figure 6 ) among the 20 examined gradients between three communities. Nonetheless, there were no significant variations in the heat index, temperature, $\mathrm{DP}, \mathrm{WB}, \mathrm{OM}, \mathrm{K}, \mathrm{P}$, and $\mathrm{CaCO}_{3}$ (Table 2).
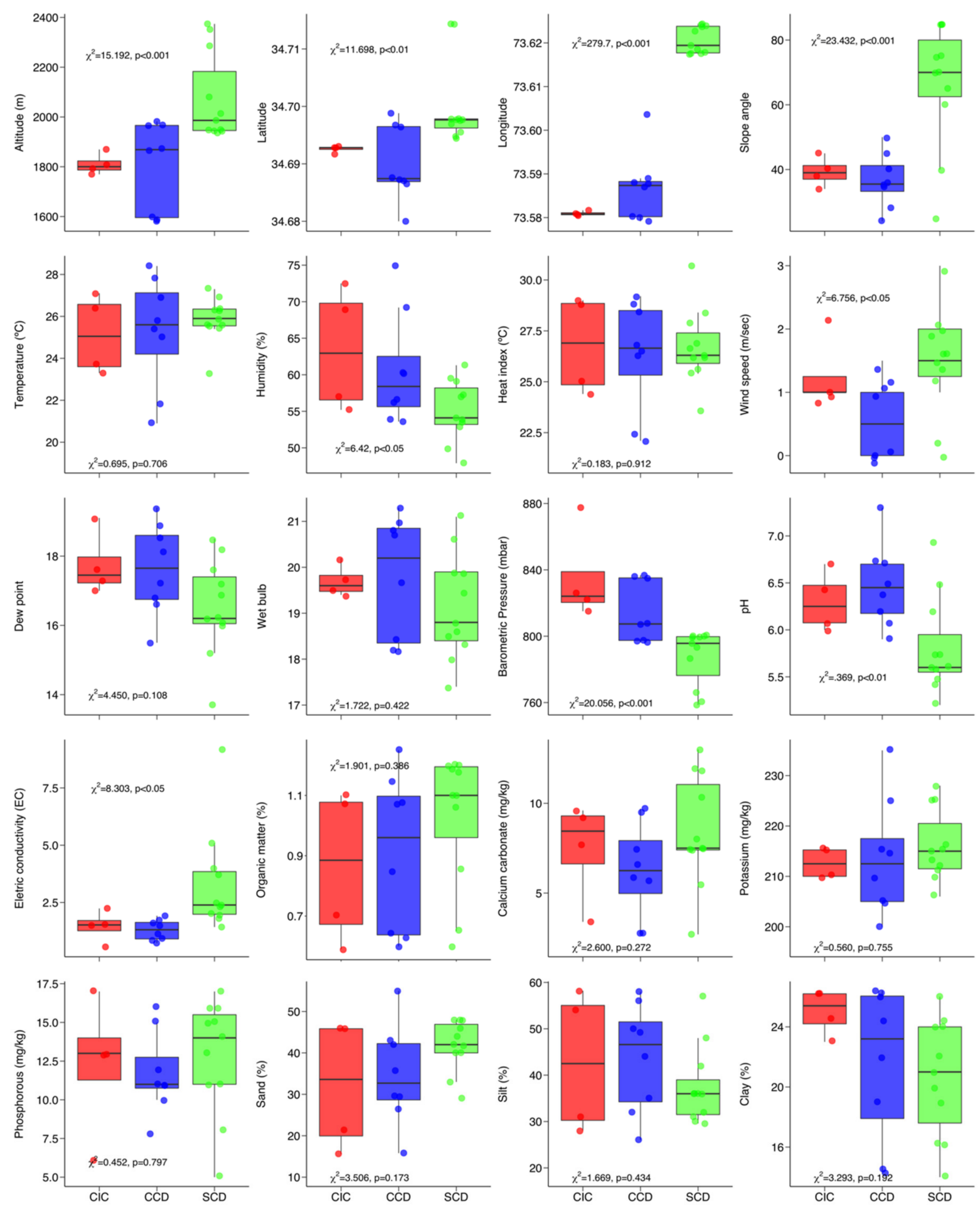

Figure 6. Variation in geographic and environmental gradients among the three plant communities of pure Deodar forests. For the complete names of plant communities, see Figure 2. 


\subsection{Beta Diversity ( $\beta$ sim and $\beta$ sne)}

Beta diversity revealed a dissimilarity lower than $50 \%$ among the three communities (Figure 7). In the $\beta$ sim cluster, we observed a $47 \%$ dissimilarity between the SCD community and the other two communities and a 15\% dissimilarity between CCD and CCI. In the $\beta$ sne cluster, the highest dissimilarity value was $28 \%$ between $\mathrm{CIC}$ and the other two communities (CCD and SCD). The CCD and SCD showed $18 \%$ dissimilarity. Thus, plant community structure is strongly influenced by $\beta$ sim as compared to $\beta$ sne, since $\beta$ sim showed almost twice the dissimilarity among communities than $\beta$ sne.
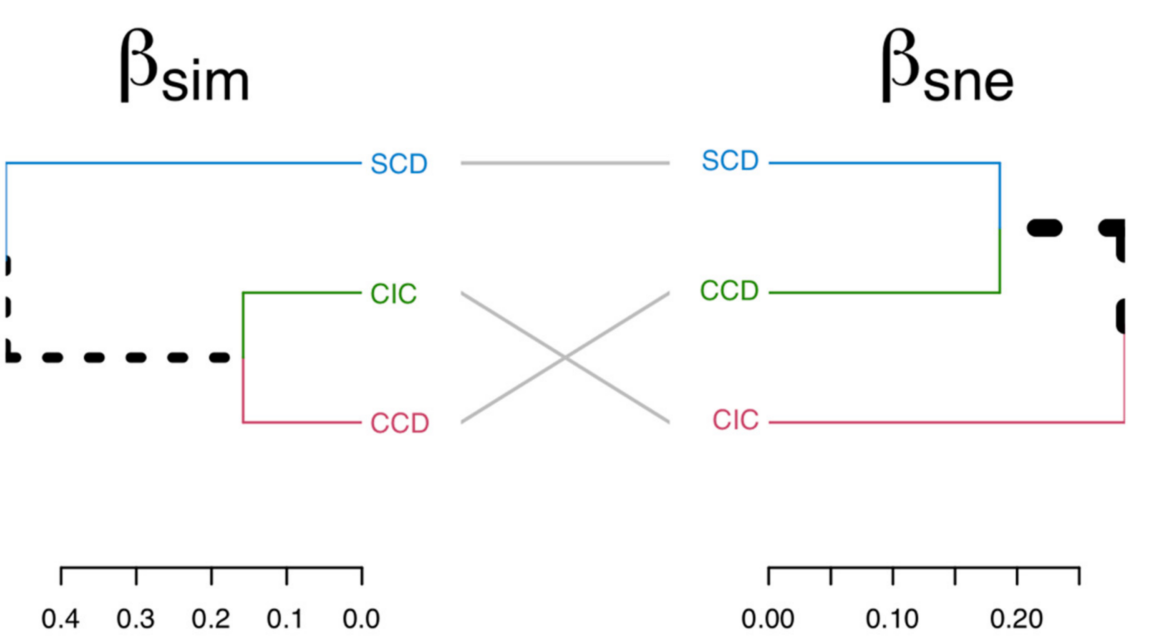

Figure 7. Beta diversity ( $\beta$ sim and $\beta$ sne) among three plant communities of pure Deodar forest. (For the complete names of plant communities, see Figure 2).

\subsection{Species Richness and Diversity Indices}

The maximum number of associated species was recorded in the SCD community (129 species, Figure 8a) at 1789.6-1896.3 m altitude, followed by CCD (66 species), and the minimum species was reported in the CIC community ( 38 species). The SCD community has the highest value $\left(\mathrm{H}^{\prime}=3.7\right)$, followed by the CCD community $\left(\mathrm{H}^{\prime}=3.6\right)$ and the CIC community $\left(\mathrm{H}^{\prime}=3.3\right.$, Figure $\left.8 \mathrm{~b}\right)$. The SCD community has the highest Simpson's dominance value (0.98), while the CIC has the lowest value (0.96, Figure $8 \mathrm{c})$. Nevertheless, the analysis revealed the CIC community with the highest value (0.97) for Pielou's evenness index, while the SCD community had the lowest value (0.93, Figure $8 \mathrm{~d}$ ).

\subsection{Variation Partitioning Tests (Partial CCA)}

The total inertia results of CCA were 3.525, where our final variables (Altitude, Temperature, Wind speed, Barometric Pressure, Slope angle, Slope ES, Slope NE, Slope SW, $\mathrm{pH}, \mathrm{EC}, \mathrm{OM}, \mathrm{CaCO}_{3}, \mathrm{~K}, \mathrm{P}$, and Silt) together explained $74.5 \%$ of the variation (eigenvalues of 2.627). The first two canonical axes explained $37 \%$ of the variation. The CCA model was significant (pseudo-F value $=2.626 ; \mathrm{df}=15 ; p<0.01$; permutations $=999$ ). For the 15 explanatory variables, we tested simple term effects. Simple term effects showed that altitude, slope angle, slope ES, and wind speed (in decreasing order of importance) were significant $(p<0.05$; Table 3$)$. All these studied gradients were further grouped into four major classes: climatic (temperature, wind speed, and barometric pressure), edaphic $(\mathrm{pH}$, $\mathrm{EC}, \mathrm{OM}, \mathrm{CaCO}_{3}, \mathrm{~K}, \mathrm{P}$, and silt), geographic (altitude), and slope (slope angle, slope ES, slope $\mathrm{NE}$, and slope SW). Moreover, variation partitioning tests (partial CCA) were performed (Table 4). Class (b) was the most explanatory variable (32.4\%), followed by class (d) $(21.1 \%)$, class (a) $(19.5 \%)$, and class (k) (17.6\%) (Figure 9$)$. 
a)

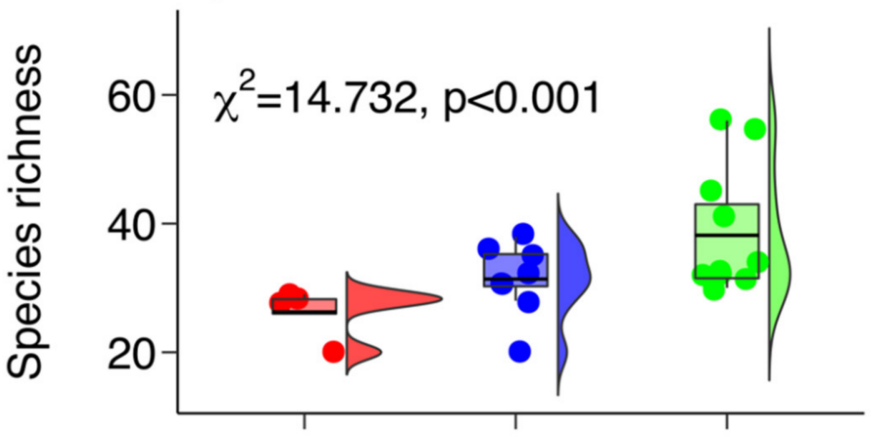

c)

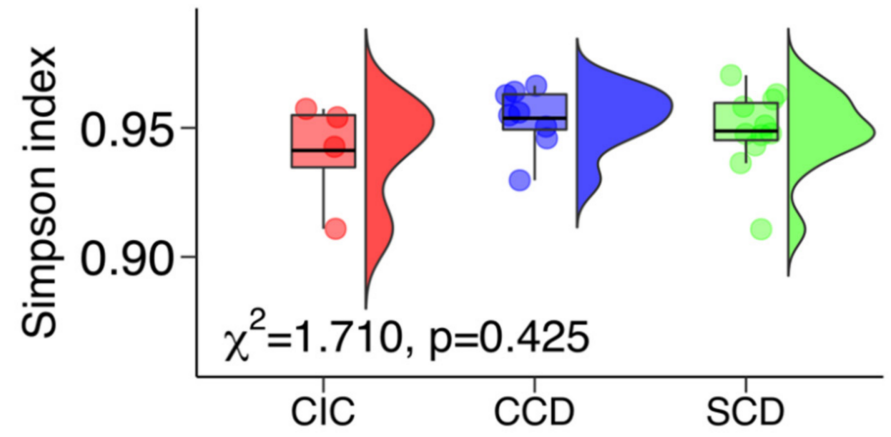

b)

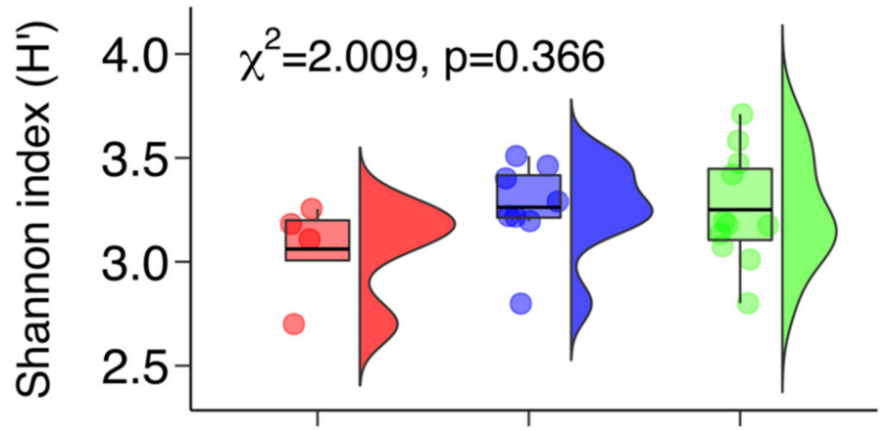

d)

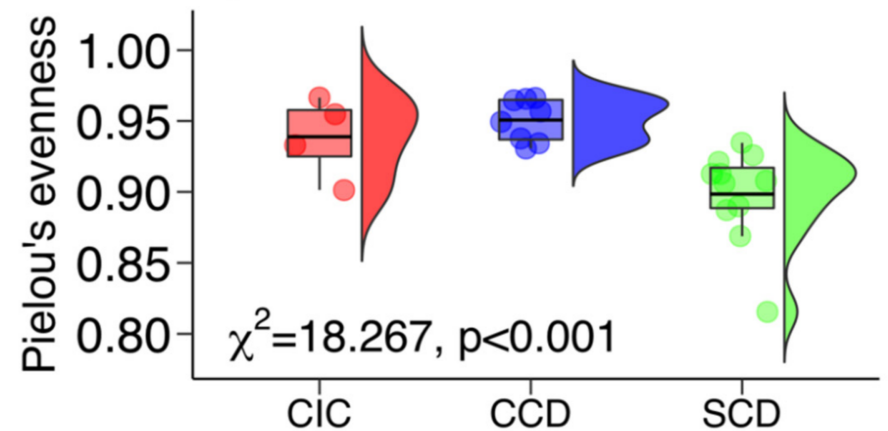

Figure 8. Variation of diversity indexes among the three plant communities recognized in pure Deodar forest (GLM results and associated $p$-values are displayed at each plot). (a-d) Species richness (a), Shannon diversity (b), Simpson diversity (c), and Pielou's evenness (d). (For the complete names of plant communities, see Figure 2).

Table 3. Contribution and ranking of the variables fit in our final model. Significant $p$-values are displayed in bold.

\begin{tabular}{ccccc}
\hline Variables & df & Chi-Square & F & Pr( $>$ F $)$ \\
\hline Altitude & 1 & 0.39074 & 30.439 & $\mathbf{0 . 0 0 1}$ \\
Temperature & 1 & 0.16637 & 12.960 & 0.144 \\
Wind speed & 1 & 0.22077 & 17.198 & $\mathbf{0 . 0 1 9}$ \\
Barometric Pressure & 1 & 0.14212 & 11.071 & 0.342 \\
Slope angle & 1 & 0.29362 & 22.874 & $\mathbf{0 . 0 0 1}$ \\
Slope (ES) & 1 & 0.25023 & 19.493 & $\mathbf{0 . 0 1 2}$ \\
Slope (NE) & 1 & 0.14662 & 11.422 & 0.290 \\
Slope (SW) & 1 & 0.17210 & 13.407 & 0.161 \\
pH & 1 & 0.10719 & 0.8350 & 0.692 \\
EC & 1 & 0.13028 & 10.149 & 0.422 \\
OM & 1 & 0.10794 & 0.8409 & 0.693 \\
CaCO & 1 & 0.13288 & 10.351 & 0.377 \\
K & 1 & 0.10015 & 0.7801 & 0.771 \\
P & 1 & 0.13604 & 10.598 & 0.343 \\
Silt & 1 & 0.12995 & 10.124 & 0.411 \\
\hline
\end{tabular}


Table 4. Variation partitioning (partial CCA) of four variable groups studied (Figure 9).

\begin{tabular}{ccccc}
\hline Individual Fraction & Adjusted R2 & Variation Explained (\%) & \% of All & df \\
\hline (a) & 0.055 & 19.5 & 1.5 & 2 \\
(b) & 0.091 & 32.4 & 2.6 & 3 \\
(c) & -0.050 & -18.0 & -1.4 & 3 \\
(d) & 0.059 & 21.1 & 1.7 & 2 \\
(e) & 0.022 & 7.8 & 0.6 & 0 \\
(f) & 0.045 & 16.1 & 1.3 & 0 \\
(g) & 0.006 & 2.2 & 0.2 & 0 \\
(h) & 0.000 & 0.1 & 0.0 & 0 \\
(i) & -0.020 & -7.3 & -0.6 & 0 \\
(j) & -0.009 & -3.3 & -0.3 & 0 \\
(k) & 0.049 & 17.6 & 1.4 & 0 \\
(l) & -0.041 & -14.8 & -1.2 & 0 \\
(m) & 0.043 & 15.4 & 1.2 & 0 \\
(n) & 0.039 & 14.0 & 1.1 & 0 \\
(o) & -0.011 & -4.0 & -0.3 & 0 \\
Total explained & 0.280 & 99.0 & 7.9 & 10 \\
All variation & 3.525 & $/$ & 100 & \\
\hline
\end{tabular}

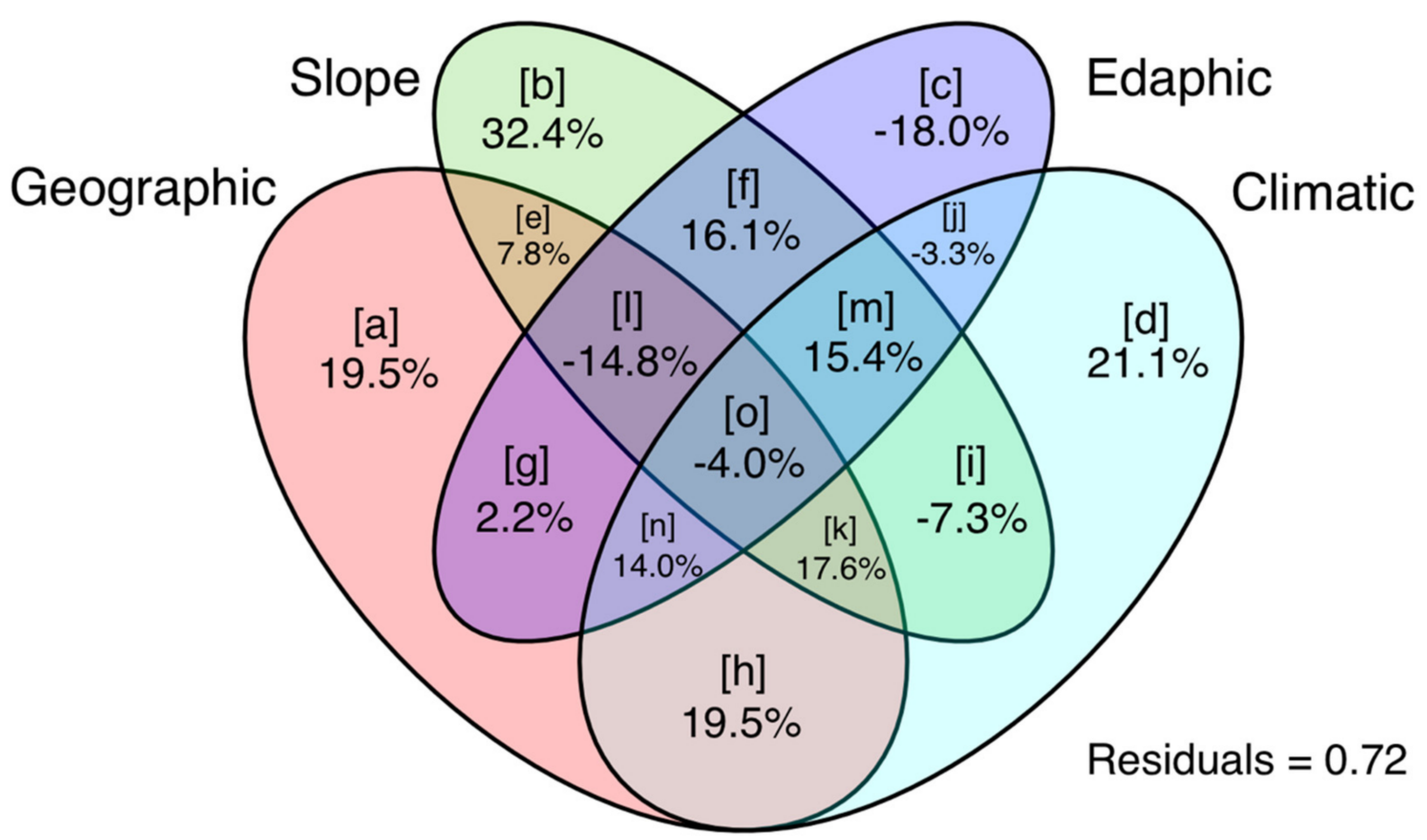

Figure 9. Venn diagram [78] illustrating the variation partitioning results (partial CCA) and contribution $(\%)$ of the four major variable classes studied.

\section{Discussion}

In this phytosociological investigation, 162 plant species were documented from 23 stands sampled in the pure Deodar forests (Manoor Valley, Himalaya, Pakistan), ranging from 1580.8 to $2373.8 \mathrm{~m}$. The species richness recorded in the current study is higher than that of temperate forests in Kedernath Wildlife Sanctuary (116 species), the Central Himalaya, India [79], and the Western Himalayan moist temperate forests in Kashmir, Pakistan (122 species) [5]. Dar and Sundarapandian [80] observed that mid-elevation (2300-2800 m) coniferous forest types had higher species richness than low and highelevation broad-leaved forests. This might be because mid-elevation ranges are less disturbed than low-elevation ranges. Although altitude is the most important variable in 
regulating species distribution, other variables, such as topography, aspect, slope, and exposure, can significantly affect the structure and composition of species $[36,81]$.

In the current ecological investigation, various types of analyses have been used to quantify the recorded data of all species and stands in association with geographic and environmental gradients. The structure and distribution patterns of plant communities in Himalayan forests are poorly understood [26]. Due to variable topographical and ecological complexity throughout a vast elevational range, the Northwestern Himalaya is a unique bioregion. The TWINSPAN, CA, and TWCA resulted in the recognition of plant communities in the pure Deodar forests of the studied area. Each plant community was composed of many stands, with no notable changes in the soil-texture values recorded for each stand. The considerable effect of the altitudinal gradient was revealed by distinguishing the vegetation of the pure Deodar forest into three major plant communities. Moreover, these three communities (Cedrus-Isodon-Cynodon, Cedrus-Cynodon-Dryopteris, and Sambucus-Cedrus-Desmodium) were established by clustering 162 species recorded from 23 stands under the effect of climatic, edaphic, topographic, and physiographic gradients in pure Deodar forest that ranged from 1580.8 to $2373.8 \mathrm{~m}$. Ahmed et al. [21] recognized seven plant communities in 47 stands of Deodar forests in the Himalayan range of Pakistan.

The SCD community showed a strong influence with altitude (1936-2373 m), slope angle (25-85), sandy (29-48\%) and loamy soil texture, wind speed $\left(1.45 \mathrm{~ms}^{-1}\right)$, and temperature $\left(25.8{ }^{\circ} \mathrm{C}\right)$. In contrast with this, the CCD community showed a positively significant relationship with the northeastern slope, silty (32-58\%) and sandy (15.8-55\%) loamy soil texture, and barometric pressure (814.3 pa). Nonetheless, the CIC community revealed a significant positive association with the northeastern to southwestern slope, $\mathrm{pH}$ (6.3), wet bulb (19.7), and dew point (17.7). A similar pattern was observed in the allied area (Nandiar catchment, Battagram) of the Himalayan region by stating altitude as the governing gradient [35]. Such variables alter the species diversity as well as community structure $[82,83]$. Species composition, community structure, and species diversity, however, are influenced not just by environmental factors but also by local micro-abiotic filtration [84].

We found significant differences $(p<0.001)$ among the three communities found in the pure Deodar forests in the four indexes. The SCD community had the maximum number of species (129 species) at 1789.6-1896.3 m altitude. Variability in the number of plant species is an outcome of species interaction with a particular set of environments [85]. A number of biological and environmental factors interact to control the distribution of species richness along the altitude [86-88]. Nonetheless, other researchers [16] reported that species richness in vascular plants increases with temperate latitude. The SCD community has the maximum Shannon's diversity $\left(\mathrm{H}^{\prime}=3.7\right)$ and Simpson's dominance $(0.98)$ values among the recorded communities. The diversity values recorded in the current study are higher than the range (1.16-3.40) reported by various researchers from other Himalayan regions $[5,89]$. The Pielou's evenness index value was led by the CIC community $(0.97)$. Many researchers reported that the physicochemical properties influence plant species richness [90] and evenness [91].

\section{Conclusions}

The current research was carried out to characterize the phytosociology of the forests, owing to one of Pakistan's most valuable tree species across its native range. In this context, this investigation documented 162 plant species recorded in 23 stands of the said forests that were further recognized into three plant communities. Each plant community was composed of many stands, with no notable changes in the soil-texture values recorded for each stand. Therefore, it could be concluded that the tree layer, rather than the soil texture, determines the structure of the ground cover. Significant differences were recorded among three communities found in the pure Deodar forests by analyzing species richness $\left(\chi^{2}=14.732, p<0.001\right)$ and Pielou $\left(\chi^{2}=18.267, p<0.001\right)$. Beta diversity showed a dissimilarity lower than $50 \%$ among the three communities. Simple term effects in the CCA model revealed significant $(p<0.05)$ differences in altitude, slope angle, slope ES, and 
wind speed variables. The present investigation sheds light on vegetation pattern and species contribution as a function of environmental gradients and provides a baseline for future studies.

Author Contributions: I.U.R., conceptualization, methodology, software, data curation, and writingoriginal draft preparation; A.A., supervision, conceptualization, and visualization; Z.I., supervision, conceptualization, methodology, and validation; E.S.C., data curation and helped in discussion; J.A., M.S.A. and N.A., manuscript editing, and discussion; R.K. and U.K., resources and helped in literature gathering; R.W.B., supervision, manuscript editing, and validation. All authors have read and agreed to the published version of the manuscript.

Funding: The authors extend their appreciation to the researchers supporting project number (RSP2021/193), King Saud University, Riyadh, Saudi Arabia.

Acknowledgments: The first author would like to express his gratitude to Pakistan's Higher Education Commission (HEC) for providing financial support to work at Missouri Botanical Garden in St. Louis, Missouri, USA. The authors extend their appreciation to the researchers supporting project number (RSP-2021/193), King Saud University, Riyadh, Saudi Arabia.

Conflicts of Interest: The authors declare no conflict of interest.

\section{References}

1. Botta-Dukát, Z.; Kovács-Láng, E.; Rédei, T.; Kertész, M.; Garadnai, J. Statistical and biological consequences of preferential sampling in phytosociology: Theoretical considerations and a case study. Folia Geobot. 2007, 42, 141-152. [CrossRef]

2. Brianskaia, E. Analysis of Phytosociological Composition and Spatial Structure of the Central Zone of Lake Baikal Eastern Coast Vegetation. Ph.D. Dissertation, Institut für Landschafts und Pflanzenökologie, Stuttgart, Germany, 2018.

3. Biondi, E. Phytosociology today: Methodological and conceptual evolution. Plant Biosyst. Int. J. Deal. All Asp. Plant Biol. 2011, 145, 19-29. [CrossRef]

4. Gairola, S.; Rawal, R.S.; Todaria, N.P. Forest vegetation patterns along an altitudinal gradient in sub-alpine zone of west Himalaya, India. Afr. J. Plant Sci. 2008, 2, 42-48.

5. Shaheen, H.; Ullah, Z.; Khan, S.M.; Harper, D.M. Species composition and community structure of western Himalayan moist temperate forests in Kashmir. For. Ecol. Manag. 2012, 278, 138-145. [CrossRef]

6. Sharma, C.M.; Ghildiyal, S.K.; Gairola, S.; Suyal, S. Vegetation structure, composition and diversity in relation to the soil characteristics of temperate mixed broad-leaved forest along an altitudinal gradient in Garhwal Himalaya. Indian J. Sci. Technol. 2009, 2, 39-45. [CrossRef]

7. Gairola, S.; Sharma, C.M.; Suyal, S.; Ghildiya, S.K. Species composition and diversity in mid-altitudinal moist temperate forests of the Western Himalaya. J. For. Environ. Sci. 2011, 27, 1-15.

8. Schmidt, I.; Zerbe, S.; Betzin, J.; Weckesser, M. An approach to the identification of indicators for forest biodiversity-The solling Mountains (NW Germany) as an example. Restor. Ecol. 2006, 14, 123-136. [CrossRef]

9. McVicar, T.R.; Körner, C. On the use of elevation, altitude, and height in the ecological and climatological literature. Oecologia 2013, 171, 335-337. [CrossRef]

10. Zhang, J.-T.; Ru, W.; Li, B. Relationships between vegetation and climate on the Loess Plateau in China. Folia Geobot. 2006, 41, 151-163. [CrossRef]

11. Pauli, H.; Gottfried, M.; Dullinger, S.; Abdaladze, O.; Akhalkatsi, M.; Alonso, J.L.B.; Coldea, G.; Dick, J.; Erschbamer, B.; Calzado, R.F.; et al. Recent Plant Diversity Changes on Europe's Mountain Summits. Science 2012, 336, 353-355. [CrossRef]

12. Iqbal, Z.; Zeb, A.; Abd-Allah, E.F.; Rahman, I.U.; Khan, S.M.; Ali, N.; Ijaz, F.; Anwar, Y.; Muzammil, S.; Alqarawi, A.A.; et al. Ecological assessment of plant communities along the edaphic and topographic gradients of Biha valley, District Swat, Pakistan. Appl. Ecol. Environ. Res. 2018, 16, 5611-5631. [CrossRef]

13. Givnish, T.J. On the causes of gradients in tropical tree diversity. J. Ecol. 1999, 87, 193-210. [CrossRef]

14. Luo, Z.; Tang, S.; Li, C.; Fang, H.; Hu, H.; Yang, J.; Ding, J.; Jiang, Z. Environmental effects on vertebrate species richness: Testing the energy, environmental stability and habitat heterogeneity hypotheses. PLoS ONE 2012, 7, e35514. [CrossRef]

15. Yang, Z.; Liu, X.; Zhou, M.; Ai, D.; Wang, G.; Wang, Y.; Chu, C.; Lundholm, J.T. The effect of environmental heterogeneity on species richness depends on community position along the environmental gradient. Sci. Rep. 2015, 5, 15723. [CrossRef]

16. Heywood, V.H.; Watson, R.T. Global Biodiversity Assessment; Cambridge University Press: Cambridge, UK, 1995 ; Volume 1140.

17. McGrady-Steed, J.; Morin, P.J. Biodiversity, density compensation, and the dynamics of populations and functional groups. Ecology 2000, 81, 361-373. [CrossRef]

18. Currie, D.J.; Francis, A.P. Regional versus climatic effect on taxon richness in angiosperms: Reply to Qian and Ricklefs. Am. Nat. 2004, 163, 780-785. [CrossRef]

19. Champion, S.H.; Seth, S.K.; Khattak, G.M. Forest types of Pakistan; Pakistan Forest Institute: Peshawar, Pakistan, 1965.

20. Hussain, F.; Illahi, I. Ecology and Vegetation of Lesser Himalayan Pakistan; Jadoon Printing Press: Peshawar, Pakistan, 1991. 
21. Ahmed, M.; Nazim, K.; Siddiqui, M.F.; Wahab, M.; Khan, N.; Khan, M.U.; Hussain, S.S. Community description of Deodar forests from Himalayan range of Pakistan. Pakistan J. Bot. 2010, 42, 3091-3102.

22. Beg, A.R. Wildlife habitats of Pakistan; Pakistan Forest Institute: Peshawar, Pakistan, 1975.

23. Ahmed, M.; Shaukat, S.S.; Siddiqui, M.F. A multivariate analysis of the vegetation of Cedrus deodara forests in Hindu Kush and Himalayan ranges of Pakistan: Evaluating the structure and dynamics. Turk. J. Bot. 2011, 35, 419-438.

24. Amin, A.; Ashfaque, R. Phytosiological studies of Ayub National Park, Rawalpindi. Pakistan J. For. 1982, 32, $130-135$.

25. Nazir, A.; Malik, R.N.; Ajaib, M. Phytosociological Studies of the vegetation of Sarsawa Hills District Kotli, Azad Jammu \& Kashmir. Biologia 2012, 58, 123-133.

26. Peer, T.; Gruber, J.P.; Millinger, A.; Hussain, F. Phytosociology, structure and diversity of the steppe vegetation in the mountains of Northern Pakistan. Phytocoenologia 2007, 37, 1-65. [CrossRef]

27. Rahman, I.U.; Hart, R.; Afzal, A.; Iqbal, Z.; Abdallah, E.F.; Alqarawi, A.A.; Ijaz, F.; Ali, N.; Kausar, R.; Muzammil, S.; et al. Phenological plasticity in Berberis lycium Royle along temporal and altitudinal gradients. Appl. Ecol. Environ. Res. 2019, 17, 331-341. [CrossRef]

28. Rahman, I.U.; Afzal, A.; Iqbal, Z.; Abd_Allah, E.F.; Alqarawi, A.A.; Calixto, E.S.; Ali, N.; Ijaz, F.; Kausar, R.; Alsubeie, M.S.; et al. Role of multivariate approaches in floristic diversity of Manoor valley (Himalayan region) Pakistan. Appl. Ecol. Environ. Res. 2019, 17, 1475-1498. [CrossRef]

29. Rahman, I.U.; Hart, R.; Afzal, A.; Iqbal, Z.; Alqarawi, A.A.; Abd_Allah, E.F.; Hashem, A.; Ijaz, F.; Ali, N.; Calixto, E.S. Ecophysiological plasticity and cold stress adaptation in Himalayan alpine herbs: Bistorta affnis and Sibbaldia procumbens. Plants 2019, 8 , 378-386. [CrossRef] [PubMed]

30. Rahman, I.U.; Afzal, A.; Iqbal, Z.; Hart, R.; Abd_Allah, E.F.; Alqarawi, A.A.; Alsubeie, M.S.; Calixto, E.S.; Ijaz, F.; Ali, N.; et al. Response of plant physiological attributes to altitudinal gradient: Plant adaptation to temperature variation in the Himalayan region. Sci. Total Environ. 2020, 706, 135714. [CrossRef]

31. Khan, S.M. Plant Communities and Vegetation Ecosystem Services in the Naran Valley, Western Himalaya. Ph.D. Dissertation, University of Leicester, Leicester, UK, 2012.

32. Ali, S.I.; Qaiser, M. A phytogeographical analysis of the phanerogams of Pakistan and Kashmir. Proc. R. Soc. Edinburgh Sect. B Biol. Sci. 1986, 89, 89-101. [CrossRef]

33. Takhtajan, A.; Crovello, T.J.; Cronquist, A. Floristic Regions of the World; University of California Press: Berkeley, CA, USA, 1986; Volume 544

34. Mustafa, G. Mansehra-An introduction. Science-Religion Dialogue Spring. 2003. Available online: https://www.hssrd.org/ journal/spring2003/mansehra.html (accessed on 27 October 2021).

35. Haq, F.; Ahmad, H.; Iqbal, Z.; Alam, M.; Aksoy, A. Multivariate approach to the classification and ordination of the forest ecosystem of Nandiar valley western Himalayas. Ecol. Indic. 2017, 80, 232-241. [CrossRef]

36. Rahman, I.U.; Afzal, A.; Iqbal, Z.; Bussmann, R.W.; Alsamadany, H.; Calixto, E.S.; Shah, G.M.; Kausar, R.; Shah, M.; Ali, N.; et al. Ecological gradients hosting plant communities in Himalayan subalpine pastures: Application of multivariate approaches to identify indicator species. Ecol. Inform. 2020, 60, 101162. [CrossRef]

37. Buckland, S.T.; Anderson, D.R.; Burnham, K.P.; Laake, J.L.; Borchers, D.L.; Thomas, L. Introduction to Distance Sampling: Estimating Abundance of Biological Populations; Oxford University Press: Oxford, UK, 2001.

38. Buckland, S.T.; Anderson, D.R.; Burnham, K.P.; Laake, J.L.; Borchers, D.L.; Thomas, L. Advanced Distance Sampling; Oxford University Press: Oxford, UK, 2004; Volume 2.

39. Buckland, S.T.; Newman, K.B.; Fernández, C.; Thomas, L.; Harwood, J. Embedding population dynamics models in inference. Stat. Sci. 2007, 22, 44-58. [CrossRef]

40. Anderson, D.R.; Burnham, K.P.; Laake, J.L. Distance Sampling: Estimating Abundance of Biological Populations; Chapman \&Hall: London, UK, 1993.

41. Le Moullec, M.; Pedersen, Å.Ø.; Yoccoz, N.G.; Aanes, R.; Tufto, J.; Hansen, B.B. Ungulate population monitoring in an open tundra landscape: Distance sampling versus total counts. Wildlife Biol. 2017, 2017, 1-11. [CrossRef]

42. Curtis, J.T.; McIntosh, R. The interrelations of certain analytic and synthetic phytosociological characters. Ecology 1950, 31, 434-455. [CrossRef]

43. Curtis, J.T. The Vegetation of Wisconsin: An Ordination of Plant Communities; University of Wisconsin Press: Madison, WI, USA, 1959; ISBN 0299019438.

44. Jayasuriya, A.H.M.; Pemadasa, M.A. Factors affecting the distribution of tree species in a dry zone montane forest in Sri Lanka. J. Ecol. 1983, 571-583. [CrossRef]

45. Son, H.-J.; Park, S.-H.; Lee, D.-H.; Song, J.-H.; Park, H.-W.; Park, W.-G. Growing environment characteristics and vegetation structure of Vaccinium Oldhamii Miq. native habitats in Korea. Forest Sci. Technol. 2019, 15, 33-43. [CrossRef]

46. Tian, Z.; Li, H.; Li, W.; Gan, X.; Zhang, X.; Fan, Z. Structural characteristics and niches of dominant tree populations in Tetracentron sinense communities: Implications for conservation. Bot. Sci. 2018, 96, 157-167. [CrossRef]

47. Lou, Y.; Pan, Y.; Gao, C.; Jiang, M.; Lu, X.; Xu, Y.J. Response of plant height, species richness and aboveground biomass to flooding gradient along vegetation zones in floodplain wetlands, Northeast China. PLoS ONE 2016, 11, e0153972. [CrossRef]

48. Song, J.H.; Cheong, E.J.; Kim, H.; Kim, M.S.; Kim, S.H. Variation of morphological characteristics and anthocyanin contents from fruit of Vaccinium oldhamii in Korea. J. Korean Soc. For. Sci. 2015, 104, 193-197. [CrossRef] 
49. Rahman, I.U.; Afzal, A.; Abd_Allah, E.F.; Iqbal, Z.; Alqarawi, A.A.; Hashem, A.; Calixto, E.S.; Ali, N.; Asmarayani, R. Composition of plant communities driven by environmental gradients in alpine pastures and cold desert of northwestern Himalaya, Pakistan. Pakistan J. Bot. 2021, 53, 655-664. [CrossRef]

50. Ijaz, F. Biodiversity and traditional uses of plants of Sarban Hills, Abbottabad. M.Phil Dissertation, Hazara University, Manehra, Pakistan, 2014.

51. Ijaz, F.; Rahman, I.; Iqbal, Z.; Alam, J.; Ali, N.; Khan, S. Ethno-ecology of the healing forests of Sarban Hills, Abbottabad, Pakistan: An economic and medicinal appraisal. In Plant and Human Health; Ozturk, M., Hakeem, K., Eds.; Springer International Publishing AG: Berlin/Heidelberg, Germany, 2018; pp. 675-706.

52. Rahman, I.U.; Afzal, A.; Iqbal, Z.; Ijaz, F.; Ali, N.; Bussmann, R.W. Traditional and ethnomedicinal dermatology practices in Pakistan. Clin. Dermatol. 2018, 36, 310-319. [CrossRef]

53. Rahman, I.U.; Ijaz, F.; Afzal, A.; Iqbal, Z.; Ali, N.; Khan, S.M. Contributions to the phytotherapies of digestive disorders: Traditional knowledge and cultural drivers of Manoor Valley, Northern Pakistan. J. Ethnopharmacol. 2016, 192, 30-52. [CrossRef]

54. Rahman, I.U.; Ijaz, F.; Iqbal, Z.; Afzal, A.; Ali, N.; Afzal, M.; Khan, M.A.; Muhammad, S.; Qadir, G.; Asif, M. A novel survey of the ethno medicinal knowledge of dental problems in Manoor Valley (Northern Himalaya), Pakistan. J. Ethnopharmacol. 2016, 194, 877-894. [CrossRef]

55. Rahman, I.U.; Afzal, A.; Iqbal, Z.; Hart, R.; Abd_Allah, E.F.; Hashem, A.; Alsayed, M.F.; Ijaz, F.; Shah, M.; Bussmann, R.W.; et al. Herbal teas and drinks: Folk medicine of the Manoor valley, Lesser Himalaya, Pakistan. Plants 2019, 8, 581. [CrossRef] [PubMed]

56. Khan, K.U.; Shah, M.; Ahmad, H.; Ashraf, M.; Rahman, I.U.; Iqbal, Z.; Khan, S.M.; Majid, A. Investigation of traditional veterinary phytomedicines used in Deosai Plateau, Pakistan. Glob. Vet 2015, 15, 381-388.

57. Nasir, E.; Ali, S.I. Flora West of Pakistan; University of Karachi, Karachi and National Herbarium: Islamabad, Pakistan, 1971.

58. Ali, S.I.; Nasir, Y.J. Flora of Pakistan; Department of Botany, University of Karachi, Karachi and National Herbarium: Islamabad, Pakistan, 1989.

59. Ali, S.I.; Qaiser, M. Flora of Pakistan; Department of Botany, University of Karachi: Karachi, Pakistan, 1995.

60. Rahman, I.U.; Ijaz, F.; Afzal, A.; Iqbal, Z. Effect of foliar application of plant mineral nutrients on the growth and yield attributes of chickpea (Cicer arietinum L.) Under nutrient deficient soil conditions. Bangladesh J. Bot. 2017, 46, 111-118.

61. Rahman, I.U.; Afzal, A.; Iqbal, Z.; Ijaz, F.; Khan, S.M.; Khan, S.A.; Shah, A.H.; Khan, K.; Ali, N. Influence of different nutrients application in nutrient deficient soil on growth and yield of onion. Bangladesh J. Bot. 2015, 44, 613-619. [CrossRef]

62. Ravindranath, N.H.; Ostwald, M. Carbon Inventory Methods: Handbook for Greenhouse Gas Inventory, Carbon Mitigation and Roundwood Production Projects; Springer International Publishing AG: Berlin/Heidelberg, Germany, 2007.

63. Mc Lean, E.O. Soil pH and Lime requirement. In Methods of Soil Analysis Part 2. Chemical and Microbiological Properties; Page, A.L., Miller, R.H., Keeney, D.R., Eds.; American Society of Agronomy, Inc./Soil Science Society of America, Inc.: Madison, WI, USA, 1982; pp. 199-223.

64. Wilson, M.J.; Bayley, S.E. Use of single versus multiple biotic communities as indicators of biological integrity in northern prairie wetlands. Ecol. Indic. 2012, 20, 187-195. [CrossRef]

65. Nelson, D.W.; Sommers, L.E. Total carbon, organic carbon, and organic matter. In Methods ofSoil Analysis. Part 3. Chemical Method; Sparks, D.L., Page, A.L., Helmke, P.A., Loeppert, R.H., Soltanpour, P.N., Tabatabai, M.A., Johnston, C.T., Summer, M.E., Eds.; Soil Science Society of America/American Society of Agronomy: Madison, WI, USA, 1996; pp. 961-1010.

66. Soltanpour, P.N. Determination of nutrient availability and elemental toxicity by AB-DTPA soil test and ICPS. In Advances in Soil Science; Springer: New York, NY, USA, 1991; pp. 165-190.

67. Smilauer, P.; Jan, L. Multivariate Analysis of Ecological Data Using CANOCO, 2nd ed.; Cambridge University Press: Cambridge, UK, 2014.

68. Mayor, J.R.; Sanders, N.J.; Classen, A.T.; Bardgett, R.D.; Clément, J.C.; Fajardo, A.; Lavorel, S.; Sundqvist, M.K.; Bahn, M.; Chisholm, C.; et al. Elevation alters ecosystem properties across temperate treelines globally. Nature 2017, 542, 91-95. [CrossRef]

69. McCune, B.; Mefford, M. PC-ORD. Multivariate Analysis of Ecological Data. Version 6; MjM Software Design: Gleneden Beach, OR, USA, 2011.

70. R Core Team. A Language and Environment for Statistical Computing; R Foundation for Statistical Computing: Vienna, Austria, 2019.

71. Bano, S.; Khan, S.M.; Alam, J.; Alqarawi, A.A.; Abd_Allah, E.F.; Ahmad, Z.; Rahman, I.U.; Ahmad, H.; Aldubise, A.; Hashem, A. Eco-Floristic studies of native plants of the Beer Hills along the Indus River in the districts Haripur and Abbottabad, Pakistan. Saudi J. Biol. Sci. 2018, 25, 801-810. [CrossRef]

72. Hill, M.O. TWINSPAN: A FORTRAN Program for Arranging Multivariate Data in an Ordered Two-Way Table by Classification of the Individuals and Attributes; Cornell University: Ithaca, NY, USA, 1979.

73. Terzi, M.; Bogdanović, S.; D'Amico, F.S.; Jasprica, N. Rare plant communities of the Vis Archipelago (Croatia). Bot. Lett. 2019, 167, 241-254. [CrossRef]

74. Smith, M.R. Create Ternary Plots in R. Available online: https://cran.r-project.org/web/packages/Ternary/vignettes/Ternary. html (accessed on 27 October 2021).

75. Fox, J.; Weisberg, S. An R Companion to Applied Regression; Sage Publications: Thousand Oaks, CA, USA, 2018.

76. Baselga, A.; Orme, C.D.L. betapart: An R package for the study of beta diversity. Methods Ecol. Evol. 2012, 3, 808-812. [CrossRef]

77. Baselga, A. Partitioning the turnover and nestedness components of beta diversity. Glob. Ecol. Biogeogr. 2010, 19, 134-143. [CrossRef] 
78. Legendre, P. Studying beta diversity: Ecological variation partitioning by multiple regression and canonical analysis. J. Plant Ecol. 2008, 1, 3-8. [CrossRef]

79. Semwal, D.P.; Uniyal, P.L.; Bhatt, A.B. Structure, composition and dominance-diversity relations in three forest types of a part of Kedarnath Wildlife Sanctuary, Central Himalaya, India. Not. Sci. Biol. 2010, 2, 128-132. [CrossRef]

80. Dar, J.A.; Sundarapandian, S. Patterns of plant diversity in seven temperate forest types of Western Himalaya, India. J. Asia-Pacif. Biodivers. 2016, 9, 280-292. [CrossRef]

81. Shank, R.E.; Noorie, E.N. Microclimate vegetation in a small valley in eastern Tennessee. Ecology 1950, 11, 531-539.

82. Adair, K.L.; Lindgreen, S.; Poole, A.M.; Young, L.M.; Bernard-Verdier, M.; Wardle, D.A.; Tylianakis, J.M. Above and belowground community strategies respond to different global change drivers. Sci. Rep. 2019, 9, 2540. [CrossRef]

83. Rahman, I.U.; Afzal, A.; Iqbal, Z.; Hashem, A.; Al-Arjani, A.-B.F.; Alqarawi, A.A.; Abd_Allah, E.F.; Abdalla, M.; Calixto, E.S.; Sakhi, S. Species Distribution Pattern and Their Contribution in Plant Community Assembly in Response to Ecological Gradients of the Ecotonal Zone in the Himalayan Region. Plants 2021, 10, 2372. [CrossRef]

84. Conti, L.; de Bello, F.; Lepš, J.; Acosta, A.T.R.; Carboni, M. Environmental gradients and micro-heterogeneity shape fine-scale plant community assembly on coastal dunes. J. Veg. Sci. 2017, 28, 762-773. [CrossRef]

85. Ricklefs, R.E. Evolutionary diversification and the origin of the diversity-environment relationship. Ecology 2006, 87, S3-S13. [CrossRef]

86. Klanderud, K.; Birks, H.J.B. Recent increases in species richness and shifts in altitudinal distributions of Norwegian mountain plants. Holocene 2003, 13, 1-6. [CrossRef]

87. Odland, A.; Birks, H.J.B. The altitudinal gradient of vascular plant richness in Aurland, western Norway. Ecography 1999, 22, 548-566. [CrossRef]

88. Weckström, J.; Korhola, A. Patterns in the distribution, composition and diversity of diatom assemblages in relation to ecoclimatic factors in Arctic Lapland. J. Biogeogr. 2001, 28, 31-45. [CrossRef]

89. Kunwar, R.M.; Sharma, S.P. Quantitative analysis of tree species in two community forests of Dolpa district, mid-west Nepal. Himal. J. Sci. 2004, 2, 23-28. [CrossRef]

90. Rahman, I.U.; Afzal, A.; Iqbal, Z.; Alzain, M.N.; Al-Arjani, A.-B.F.; Alqarawi, A.A.; Abd_Allah, E.F.; Ali, N.; Sakhi, S.; Khan, M.A.; et al. Classification and Characterization of the Manoor Valley's (Lesser Himalaya) Vegetation from the SubtropicalTemperate Ecotonal Forests to the Alpine Pastures along Ecological Variables. Plants 2022, 11, 87. [CrossRef] [PubMed]

91. Oduor, C.; Karanja, N.; Onwonga, R.; Mureithi, S.; Pelster, D.; Nyberg, G. Enhancing soil organic carbon, particulate organic carbon and microbial biomass in semi-arid rangeland using pasture enclosures. BMC Ecol. 2018, 18, 45. [CrossRef] [PubMed] 